\title{
The interaction of two co-rotating quasi-geostrophic vortices in the vicinity of a surface buoyancy filament
}

\author{
JEAN N. REINAUD†* \\ † Mathematical Institute, North Haugh, St Andrews, KY169SS, UK \\ (14 September 2017)
}

\begin{abstract}
In this paper, we investigate the interaction between two like-signed quasi-geostrophic uniform potential vorticity internal vortices in the vicinity of a surface buoyancy anomaly filament in a three dimensional, stably stratified and rapidly rotating fluid. The surface buoyancy distribution locally modifies the pressure fields and generates a shear flow. We start the study by first considering the effects of a uniform linear horizontal shear on the binary vortex interaction. We confirm that a cooperative shear facilitates the merger of a pair of vortices while an adverse shear has the opposite effect. We next investigate the binary vortex interaction in the vicinity of the surface buoyancy filament explicitly. Here, not only the filament generates a shear flow, but it also responds dynamically to the forcing by the vortex pair. The filament destabilises and forms buoyancy billows at the surface. These billows interact with the internal vortices. In particular, a surface billow may pair with one of the internal vortices. In such cases, the like-signed internal vortex pair may separate if they are initially moderately distant from each other.
\end{abstract}

Keywords: Vortex dynamics; Vortex merger; Surface quasi-geostrophy; Quasi-geostrophy

\section{Introduction}

Vortices are key dynamical features of the oceans. They are known from observational evidences such as satellite imagery to carpet the surface layers of the oceans. Vortices are also found at finite depths. A well known example of such internal vortices is the 'Meddies' (Mediterranean Water Eddies), see for example Richardson, McCartney and Maillard (1991) and Richardson, Bower and Zenk (2000). These eddies are generated by the destabilisation of the Mediterranean outflow in the North Atlantic ocean. They typically lie at a depth of order of $1000 \mathrm{~m}$, where the salty Mediterranean 'surface' waters becomes buoyant in the Atlantic waters.

Collectively mesoscale vortices contribute to at least half of the transport of heat, momentum and tracers in the oceans according to recent estimates, see Zhang, Wang and Qiu (2014). Hence the study of the elementary mechanisms of their mutual interaction and of their interaction with their surroundings is essential to acquire a better theoretical understanding of ocean dynamics.

Like-signed vortices sometimes merge together to form larger coherent structures. Vortex merger has been suggested as one of the mechanisms to explain in physical space the transfer of energy towards large scales observed for example in spectral space in two-dimensional turbulence. Vortex merger is also normally associated with the shedding of vorticity filaments and small debris as a consequence of the conservation of invariants, in particular the angular impulse, contributing to the direct enstrophy cascade. Vortex merger has first been extensively studied in the literature, first in two dimensional flow, see Overman,II and Zabusky (1982), Melander, Zabusky and McWilliams (1988), Dritschel and Waugh (1992), Waugh (1992),

\footnotetext{
*Corresponding author: Email: jean.reinaud@st-andrews.ac.uk
} 
Dritschel (1995) to name but a few studies. One of the outcomes of these studies is that two identical two-dimensional uniform vortices merge provided they are separated by a distance $d<3.3 R$, where $R$ is the vortices radius. Additionally, the merger of two-dimensional vortices in a linear shear has been studied by Trieling, Dam, and van Heijst (2010). The authors concluded that a cooperative shear enhanced the possibility of merger while vortices in adverse shear may separate. Similar conclusions are reached here in our study for the merger of three dimensional quasi-geostrophic vortices. Note that Carton, Maze and Legras (2002) also studied the merger of two dimensional vortices under external strain.

The merger of two like-signed three-dimensional quasi-geostrophic vortices has been studied in von Hardenberg at al. (2000), Dritschel(2002) for identical vortices, Reinaud and Dritschel (2002) for identical but vertically offset vortices, Reinaud and Dritschel (2005), Bambrey, Reinaud and Dritschel (2007), Ozugurlu, Reinaud and Dritschel (2008) for vortices of different volumes and/or strengths. Again, three dimensional quasi-geostrophic vortices may merge provided they are separated by less than a critical merging distance. This distance is typically less than the one in a equivalent two dimensional case as the velocity induced by a vorticity source decays more rapidly in three dimensions than it does in two dimensions. Indeed, the induced velocity falls as $r^{-2}$ in a three dimensional flow, where $r$ is the distance between the vorticity source and the evaluation point, whereas it falls as $r^{-1}$ in a two dimensional flow.

On the other hand, the interaction of isolated vortices with a surface buoyancy filament has been studied in Reinaud, Dritschel and Carton (2016), while the interaction of a heton with the filament is presented in Reinaud, Carton and Dritschel (2017). A heton consists of two vortices of opposite sign lying at different depths. It should be noted that a filament of buoyancy is unstable, see Juckes (1995), Harvey and Ambaum (2010), and Reinaud, Dritschel and Carton (2016). In both studies by Reinaud et al. $(2016,2017)$, the dynamical coupling of an internal vortex with a surface billow formed by the destabilisation of the surface buoyancy filament is often observed. Similar couplings are also observed for a single vortex or a vortex dipole interacting with a surface buoyancy jet, see Reinaud, Dritschel and Carton (2017).

The merger of two cyclonic vortices in the vicinity of the Azores current has been observed during the SEMAPHORE ("Structure des Echanges Mer-Atmosphere, Propriétés des Hétérogénéites Océaniques: Recherche Expérimentale") experiment of 1993-1995 , see Tychensky and Carton (1988), indicating that the study of vortex merger in the vicinity of a shear zone is of practical and theoretical interest. The present contribution does not aim to reproduce the situation observed during the SEMAPHORE experiment. Instead, we focus here on the elementary interactions between a pair of like-signed vortices and a surface buoyancy filament, known to be commonly observed on the ocean surface, see Iermano et al. (2012), Gula et al. (2014), McWilliams et al. (2014). The filament is used as a feature to generate a shear flow at the surface. Filament may be generated by several different physical mechanisms: instabilities at upwelling fronts, interactions between coastal currents and topography, vorticity transfer between the wind field and the sea, see Iermano et al. (2012). Filaments typically have a few days life time, up to 40-50 days in Haidvogel et al. (1991), while Meddies can persist for a couple of years. Most of the existing studies on filament formation and dynamics have focused on coastal regions.

The paper is organised as follows. Section 2 describes the mathematical and numerical setups. Before investigating the details of the interaction between the pair of internal like-signed vortices and the surface filament, the influence of a uniform (fixed) background shear on the interaction between two liked-signed vortices is revisited in Section 3. We confirm that a cooperative shear enables the vortices to merge from further apart, while an adverse shear tends to separate the vortices. An appendix presenting a simplified model of the behaviour of a point vortex pair under uniform shear is included at the end of the paper. Section 4 is dedicated to the study of the vortex merger in the vicinity of the filament. Here, we show the additional influence of the coherent structures which form at the surface as a result of the 
destabilisation of the shear zone. Finally, we present some conclusions in Section 5.

\section{Mathematical model}

We consider a three dimensional, stably stratified flow subject to a rapid background rotation for which the Rossby number, $\operatorname{Ro}=U /(f L) \ll 1$, and the Froude number $F r=U /(N H) \ll$ $R o^{1 / 2}$. Here $U$ is a typical horizontal velocity scale, $f$ is the Coriolis frequency quantifying the planet's rotation, $L$ is a typical horizontal length scale, $H$ is a typical vertical length scale, and $N$ is the buoyancy or Brunt-Väisälä frequency quantifying the effects of the density stratification. More specifically $N$ is the oscillation frequency of the fluid parcel displaced in the vertical from its equilibrium position in absence of rotation (or in the short horizontal wave limit). For simplicity, we take both $f$ and $N$ constant and we conveniently stretch the vertical coordinate by the constant ratio $N / f$. In practice $N / f \sim 10-100$ over wide areas of the ocean, see for example Dijkstra (2008). In this regime, the primitive equations can be asymptotically expanded to obtain the Quasi-Geostrophic (QG) model, see Vallis (2006) for a full derivation. This model is arguably the simplest dynamical model relevant to flows under rapid background rotation in a stable stratification, and yet it remains very well suited to model mesoscale ocean dynamics.

The flow is fully described by a single prognostic variable, the potential vorticity, hereinafter referred to as $\mathrm{PV}, q$ (or the buoyancy $b$ at the surface) which is materially conserved in absence of frictional or diabatic effects,

$$
\frac{\mathrm{D} q}{\mathrm{D} t}=0, \text { and } \frac{\mathrm{D} b}{\mathrm{D} t}=0 .
$$

Here $\mathrm{D} / \mathrm{D} t=\partial / \partial t+u \partial / \partial x+v \partial / \partial y$ is the material derivative. Note that the incompressible advecting velocity field is layerwise two-dimensional as the vertical velocity is negligible. The velocity field can be obtained from the potential vorticity (and buoyancy) by linear inversion relations. The scalar streamfunction is conveniently split into two parts,

$$
\psi=\psi_{i}+\psi_{s},
$$

where $\psi_{i}$ is the part of the streamfunction induced by the (interior) PV $q$, and $\psi_{s}$ is the part of the streamfunction induced by the surface buoyancy $b$. The inversion relations read

$$
\Delta \psi_{i}=q, \quad \Delta \psi_{s}=0,
$$

where $\Delta$ is the three-dimensional Laplace's operator in the vertically stretched coordinate system. The domain is doubly periodic in the horizontal directions with, without loss of generality, horizontal dimension $[-\pi, \pi]^{2}$. For the investigation of the interaction of the vortex pair and the surface buoyancy filament in Section 4 , the vertical boundary conditions consist of a flat impermeable surface at the ocean bottom $\left(z=-H_{b}=-2 \pi\right)$ and are set by the surface buoyancy distribution at the ocean surface $(z=0)$, see Perrot et al. (2010), Reinaud, Dritschel and Carton $(2016,2017)$ and Reinaud, Carton and Dritschel (2017) for similar set-ups. These conditions are mathematically expressed as follows

$$
\begin{aligned}
& \frac{\partial \psi_{s}}{\partial z}\left(x, y,-H_{b}\right)=0, \quad \frac{\partial \psi_{s}}{\partial z}(x, y, 0)=\frac{b}{N}=b^{*}, \\
& \frac{\partial \psi_{i}}{\partial z}\left(x, y,-H_{b}\right)=0, \quad \frac{\partial \psi_{i}}{\partial z}(x, y, 0)=0 .
\end{aligned}
$$

The velocity is given by 


$$
\boldsymbol{u}=\boldsymbol{\nabla} \times \psi \boldsymbol{k}=\left(-\frac{\partial \psi}{\partial y}, \frac{\partial \psi}{\partial x}, 0\right),
$$

where $\boldsymbol{k}$ is the vertical unit vector pointing upwards.

The equations are solved numerically using the Contour-Advective Semi-Lagrangian (CASL) algorithm, developed by Dritschel and Ambaum (1997), and extended to the three-dimensional QG equations including variable surface buoyancy by Perrot et al. (2010). The inversion relations are solved on a $256^{3}$ grid in the interior, and on a grid four times finer with a resolution of $1024^{2}$ at the surface as finer scales are expected to be generated at the surface, see Juckes (1995), Scott (2011), Scott and Dritschel (2014). The interior of the domain is mapped by 1024 layers. The resolution of the contours (or jumps) describing the prognostic fields is controlled by the contour surgery technique introduced in Dritschel (1988), with a 'surgery' scale $\delta_{s}$ set to one sixteenth of the inversion grid length, i.e. $\delta_{s}=2 \pi / 4096$. The equations are marched in time using a fourth-order Runge-Kutta integration scheme, with a time step $\Delta t$ controlled by local stretching of the buoyancy contours or the PV time scale, whichever criterion dictates the smallest time step. Specifically, $\Delta t=\min \left(\pi /(20 q), 0.2 / \max _{i}\left\{s_{i}\right\}\right)$, where $s_{i}=\left\|\boldsymbol{u}_{i+1}-\boldsymbol{u}_{i}\right\| /\left\|\boldsymbol{x}_{i+1}-\boldsymbol{x}_{i}\right\|$ is the contour stretching rate between two adjacent nodes $i$ and $i+1$ on a contour.

\section{Vortex merger under uniform shear}

In this section, the surface buoyancy is set to zero, and the interior PV is inverted on a triply periodic domain of size $[-\pi, \pi]^{3}$ for simplicity. Two vortices are initially spheres ${ }^{1}$ of uniform $\mathrm{PV}, q=2 \pi$, and of radius $R=0.25$. The vortices are initially located at $(0, \pm d / 2,0)$ such that they are initially separated by a distance $d_{v}(t=0)=d$ in the $y$-direction. The vortices are small compared to the domain size, and the effects of the periodic images are limited. The vortices rotates around one another. This period of rotation $T_{\text {rot }}$ can be estimated by modelling each vortex by a singularity carrying the volume integral of $\mathrm{PV} \kappa=\iiint_{v} q d v=$ $4 \pi q R^{3} / 3$. The rotation rate for pair of point vortices is given by $\Omega=\frac{1}{4 \pi} \frac{2 \kappa}{d^{3}}=\frac{2 q}{3(d / R)^{3}}$, hence $T_{\text {rot }}=\frac{2 \pi}{\Omega}=3(d / R)^{3} / 2$ here.

Using the linearity of the inversion operator (a Laplacian), an external linear shear is simply added to the velocity field. Note that the shear itself is not periodic and is not obtained by inversion. But it simply consists in a fixed velocity field in the $y$-direction $v_{s}=\alpha x$. We define $\lambda_{s}=\alpha / q$ the non dimensional constant ratio of the uniform background shear to the vortices $\mathrm{PV}$. The equations are solved using the triply periodic CASL on a $256^{3}$ inversion grid while, again, the domain is mapped by 1024 layers. The simulations are run until $T=30$ which corresponds to 10 turnover periods $T_{\text {over }}$ for the vortices. Recall that an isolated sphere of uniform PV $q$ has a rotation period of $T_{\text {over }}=6 \pi / q$.

We perform a large number of simulations for various values of initial relative distance between the vortices $d / R$ and the shear ratio $\lambda_{s}$. Note that the background shear corresponds to a (vertical) vorticity $\zeta_{s}=\partial v_{s} / \partial x=q \lambda_{s}$. Hence, $\lambda_{s}>0$ corresponds to a cooperative shear while $\lambda_{s}<0$ corresponds to an adverse shear. We first classify the outcome of the interactions by sorting them in 10 categories. To that purpose we diagnose in particular two characteristics of the vortices. The two vortices are first best-fitted to ellipsoids with centroid $\boldsymbol{X}_{i=1,2}$, and axis semi-lengths $\left(a_{i}, b_{i}, c_{i}\right)_{i=1,2}$ where $c_{i} \leq b_{i} \leq a_{i}$, and we monitor $(i)$ the time dependent

${ }^{1}$ Note that the vortices are only spheres in the vertically stretched coordinate system. They are pancake vortices in physical space. 


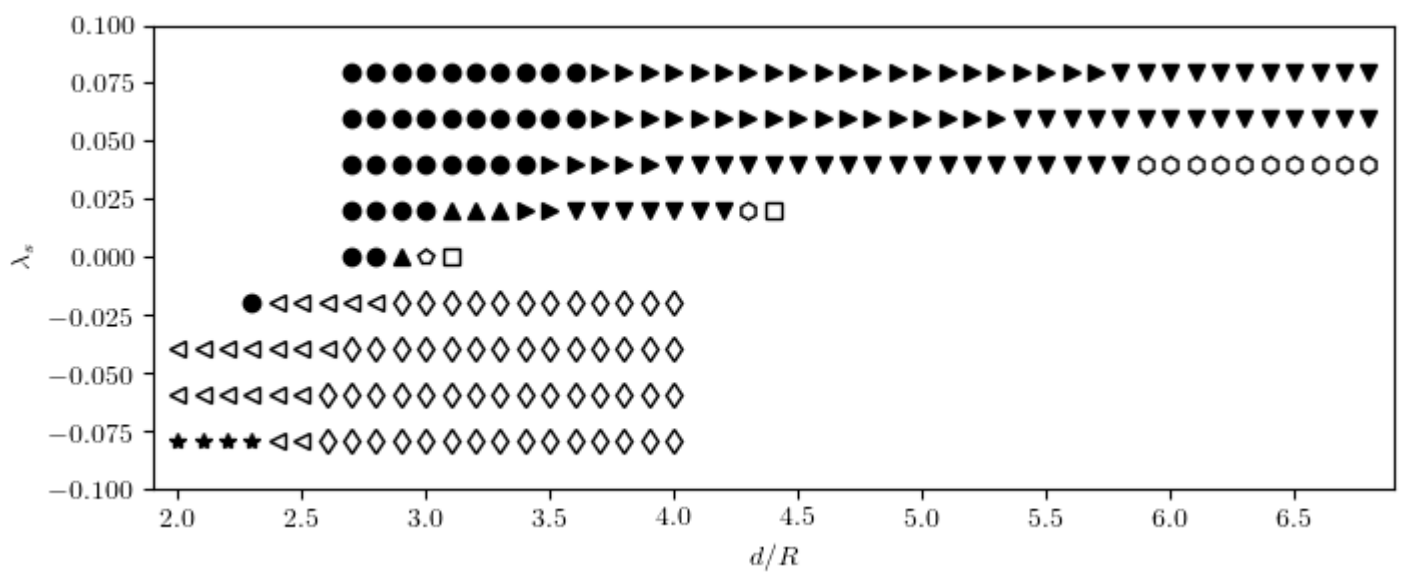

Figure 1. Outcome of the interaction between two spherical vortices of radius $R$, and PV $q$, initially distant of $d$, under a uniform horizontal shear $v=\lambda_{s} q x$. • corresponds to Merger, $\square$ to No Merger, $\longrightarrow$ to Late Merger, $\boldsymbol{\Delta}$ to Merger/Break/Merger, $\boldsymbol{\nabla}$ to Late Merger/Break/Merger, $\diamond$ to Late Merger/Break, $\diamond$ to Separation, $\triangleleft$ to Merger/Break/Separation, $\star$ to Merger/Shear and $\square$ to Touch/Break.

distance $d_{v}(t)=\left|\boldsymbol{X}_{2}-\boldsymbol{X}_{1}\right|$ between the vortices centroids and (ii) their aspect ratio $c / a$. Note that by symmetry the aspect ratios of the two vortices are roughly the same. The results are summarised in figure 1 , and the categories are as follows:

(i) Merger (a • in figure 1). The two vortices deform by elongating and merge together directly.

(ii) No Merger (a $\square$ in figure 1). The vortices do not merge and the distance between the vortices $d_{v}$ remains within $10 \%$ of its initial value $d$.

(iii) Late Merger (a in figure 1). Similar to the Merger, but the vortices aspect ratio $c_{i} / a_{i}$ oscillates before the vortices merge.

(iv) Merger/Break/Merger (a $\boldsymbol{\Delta}$ in figure 1). The vortices merge directly. The merged structure breaks back to two individual vortices which eventually merge again.

(v) Late Merger/Break/Merger (a $\boldsymbol{\nabla}$ in figure 1). Same as above, but the first merger is a Late Merger.

(vi) Late Merger/Break (a $\oslash$ in figure 1). The vortices merge after oscillations but eventually break.

(vii) Separation (a $\diamond$ in figure 1). The vortices do not merge and their separation distance $d_{v}$ increases with time.

(viii) Late Merger/Break/Separation (a $\triangleleft$ in figure 1). The vortices first merge, then the structure break back into two individual vortices which are being separated by the background flow.

(ix) Merger/Shear (a $\star$ in figure 1). The vortices merge, and then the merged structure is sheared (elongated) by the background shear flow.

(x) Touch/Break (a $\square$ in figure 1). The vortices periodically touch to form a temporary single (connected) structure which breaks back into individual vortices.

Figure 1 shows clearly that the external background shear has a strong influence on the interaction, with a clear contrast between the cooperative and adverse shear cases. In the absence of external shear, the vortices merge for a critical separation distance $d_{M} / R \leq 2.8$. Note from figure 1 that the minimum increment in separation distance between two cases is 0.1 , hence all the values for critical distances have to be understood as accurate within \pm 0.1 . The addition of a cooperative external shear increases this critical distance from $d_{M} / R \simeq 3$ for $\lambda_{s}=0.02$ up to $d_{M} / R \simeq 3.7$ for $\lambda_{s}=0.08$ which corresponds to an increase for the distance 

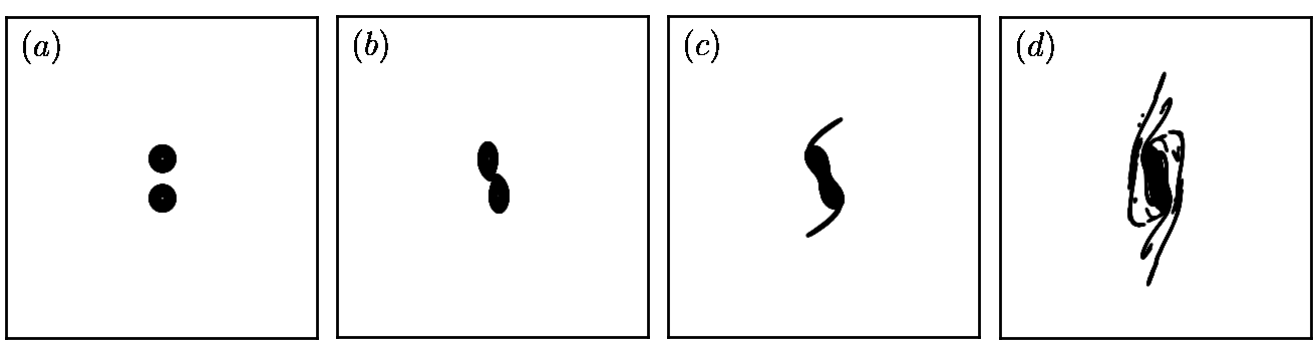

Figure 2. Illustration of a Merger ( $\bullet$ in figure 1): Top view on the PV contours defining the vortices boundary for $d / R=3.1$, and $\lambda_{s}=0.04$ displayed at $t=0(a), 1.8(b), 10(c)$, and $30(d)$.
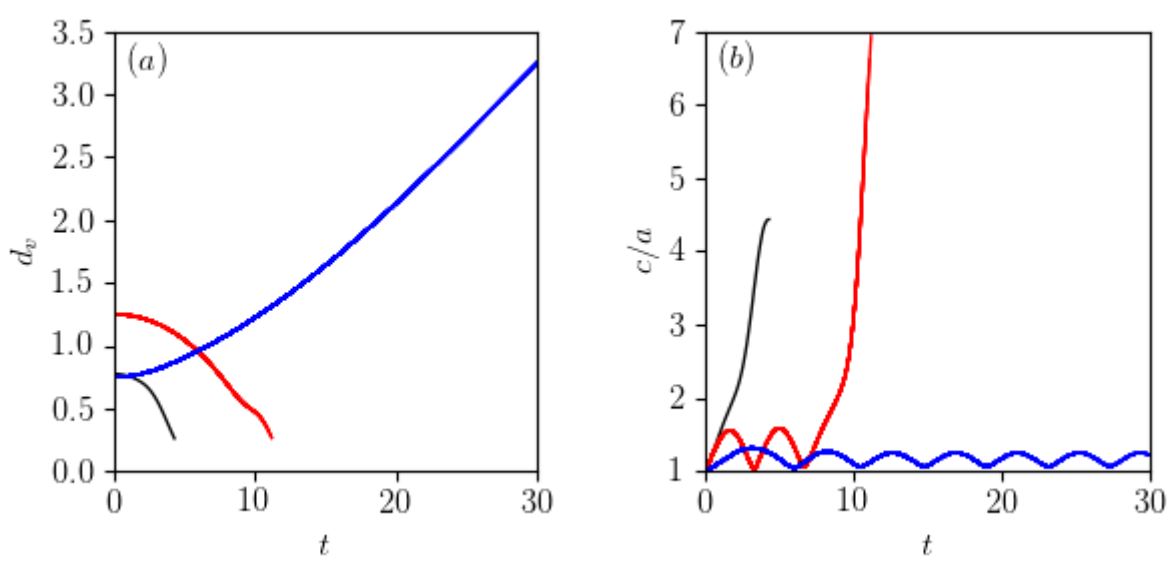

Figure 3. (a) Distance $d_{v}$ between the vortices centroids vs time for $d / R=3.1, \lambda_{s}=0.04$ (in black, the Merger illustrated in figure 2), $d / R=5, \lambda_{s}=0.06$ (in red, the Late Merger illustrated in figure 4), and $d / R=3, \lambda_{s}=-0.02$ (in blue, the Separation illustrated in figure 8). (b) vortex aspect ratio $c_{1} / a_{1}$ vs time for the same cases (colour online).

of $32 \%$ for a shear amounting for $8 \%$ of vortex PV for the latter case when compared to the case without shear $\lambda_{s}=0$.

An example of merger is proposed in figure 2 for $d / R=3.1$ and $\lambda_{s}=0.04$. This example is representative of all the merger cases investigated. The vortices continuously elongate developing sharp inner edges. These edges do not align along the axis joining the vortex centroids but are slightly offset, and the vortices merge from the side adjacent to the inner edges. These features are typical of the classical vortex merger. The evolution of the distance measured between the two vortex centroids is given in figure 3(a) (black curve) and it shows the rapid convergence of the two centres. The curve stops when the two vortices have merged into a single vortex at $t=4.3$. Figure 3(b) (black curve) shows the evolution of one of the vortices aspect ratio $c / a$. Recall that by symmetry the vortices share the same aspect ratio within the precision of the calculation. It confirms that the vortex monotonically elongates until the merger.

For a cooperative shear, $\lambda_{s}>0$, increasing the relative separation distance $d / R$ from the critical merger value $d_{M} / R$ first results in a late merger in which the vortices do not merge at once but first undergo an initial phase where the vortices periodically pulse (their aspect ratio $c_{i} / a_{i}$ oscillates in time) and the vortices slowly come closer together. When the vortices are close enough, the inviscid merger process starts. An example of late merger is presented in figure 4 for $d / R=5$, and $\lambda_{s}=0.06$. This example is representative of all late merger cases. The first three panels illustrate the oscillation of the vortices aspect ratio, and the decrease in their separation distance is noticeable. The later merger process itself is very similar to the classical one. Figure 3(b) (red curve) illustrates clearly two quasi-periodic deformation of 

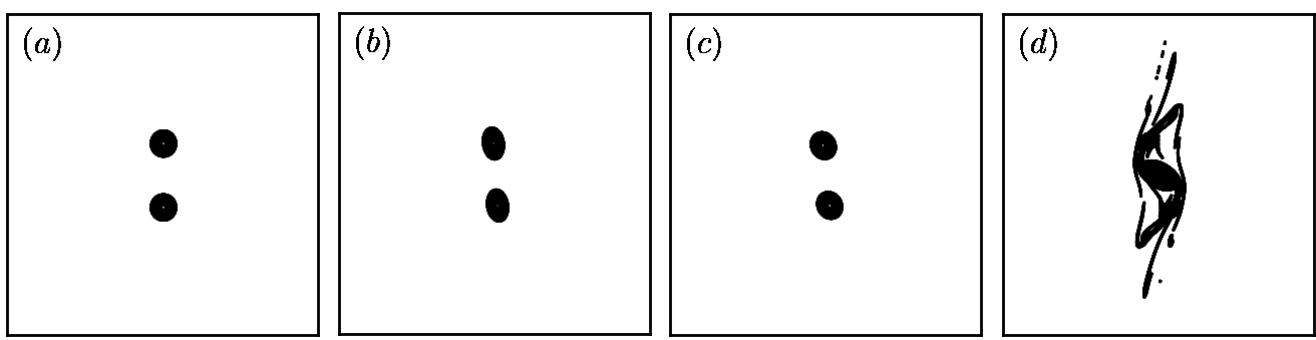

Figure 4. Illustration of a Late Merger $(\checkmark$ in figure 1): Top view on the PV contours defining the vortices boundary for $d / R=5$, and $\lambda_{s}=0.06$ displayed at $t=0(a), 2(b), 3(c)$, and $30(d)$.
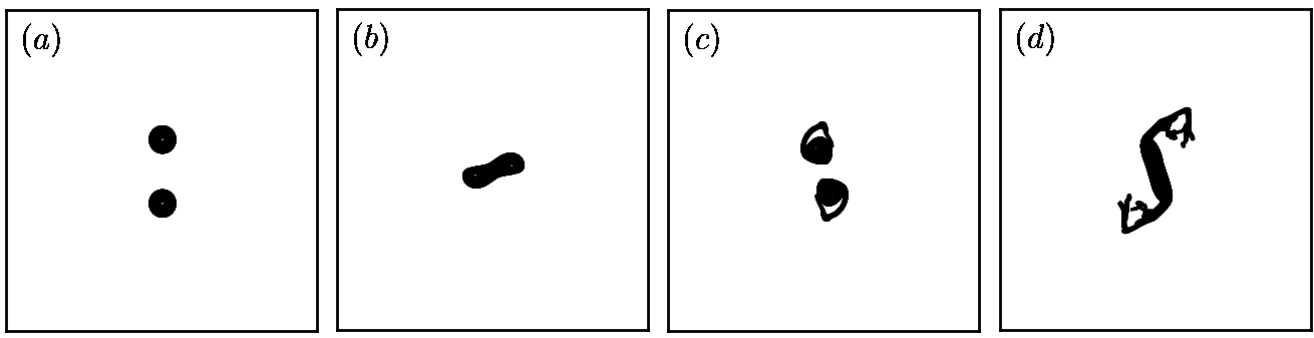

Figure 5. Illustration of a Late Merger/Break/Merger ( $\mathbf{v}$ in figure 1): Top view on the PV contours defining the vortices boundary for $d / R=5$, and $\lambda_{s}=0.04$ displayed at $t=0(a), 2(b), 21(c)$, and $30(d)$.

the vortices at early stage. The later continuous elongation starts from $t=3.4$. At this stage, $d_{v} / R \simeq 4.6$. By the time of merger, the distance between the vortex centroids is roughly the same as in the previous merger case illustrated, but the vortices are much more elongated. The reason may be that the process started from vortices further apart and the shear (which is larger) had more time to elongate the structures. It should also be noticed that in both cases the merger produces filaments which are further stretched by the external shear.

For even larger initial separation distances, the vortices can still merge but the merged structure is an elongated dumbbell-like structure which breaks back into individual vortices. The vortices are pushed further together by the external shear and eventually merge back. A typical example, representative of all such cases is proposed in figure 5 for $d / R=5$, and $\lambda_{s}=0.04$. By $t=6$, the two vortices have merged creating a unstable dumbbell structure which later breaks. Panel $(c)$ of figure 5 shows the quasi-symmetric vortices produced by the splitting of the dumbbell vortex. The vortices are shedding filaments. Eventually these structures merge again and more PV is fed to the filaments.

Increasing further the initial separation distance results in the inability of the split dumbbell structure to merge back, at least during the simulation time window of our simulations, $t \in[0,30]$.

Finally, for small positive value of the shear, the vortices no longer merge for large separation distances $\left(d / R \geq 4.5\right.$ for $\left.\lambda_{s}=0.02\right)$. This regime was not observed though for $\lambda_{s} \geq 0.04$ and $d / R \leq 7$. For $d / R \geq 7$ the vortices already occupy a large part of the domain and the period images may start to have a influence on the dynamics. Indeed, the outer edges of the vortices are distant of $\ell=9 R=2.25$ at $t=0$ which corresponds to $35.8 \%$ of the domain length. Consequently we have not studied the interaction for larger initial separation distances. Note that overall the critical separation distances between the different regimes are increased as the shear is increased.

The regimes for adverse shear, $\lambda_{s}<0$, are different. For the small value (in magnitude) of adverse shear, $\lambda_{s}=-0.02$, the vortices merge for $d / R \leq 2.4$. This is significantly less than the critical merging distance for the isolated case with $\lambda_{s}=0$. For the same value of $\lambda_{s}$, and $2.5 \leq d / R \leq 2.8$ the vortices first merge, but the merged dumbbell structure is sheared by the 

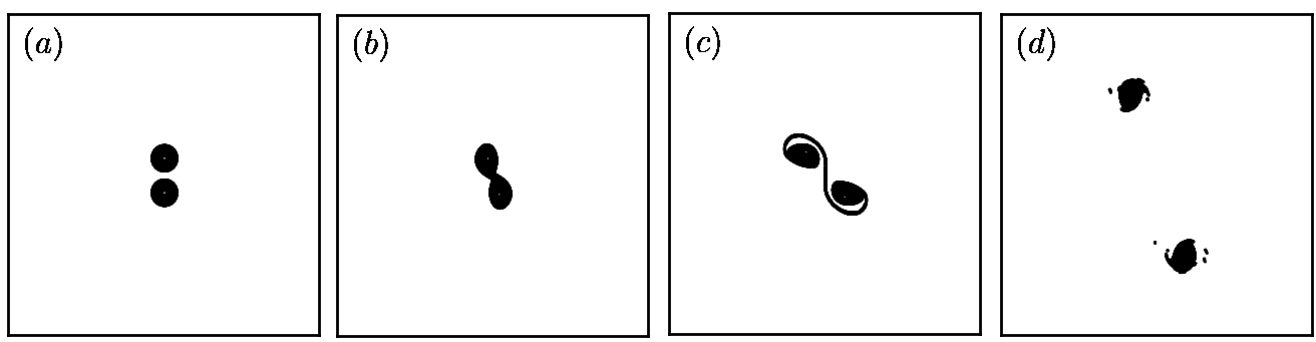

Figure 6. Illustration of a Merger/Break/Separation ( $\triangleleft$ in figure 1): Top view on the PV contours defining the vortices boundary for $d / R=2.7$, and $\lambda_{s}=-0.02$ displayed at $t=0(a), 1.8(b), 10(c)$, and $30(d)$.
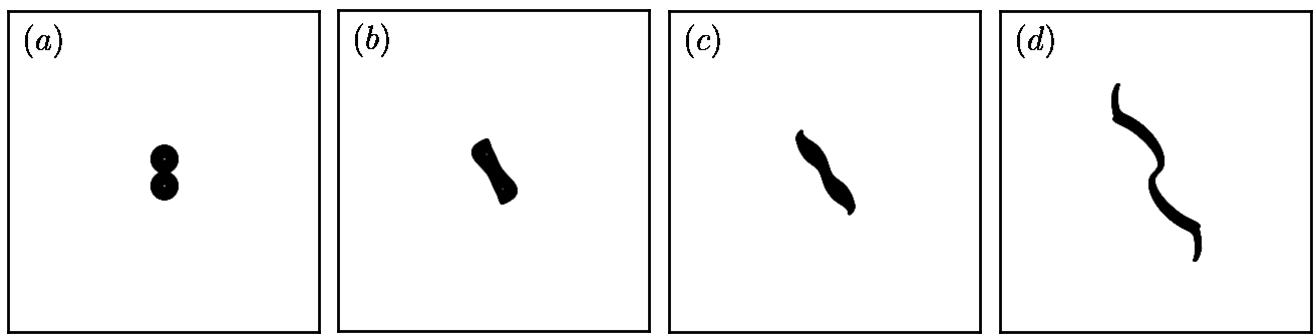

Figure 7. Illustration of a Merger/Shear ( $\star$ in figure 1): Top view on the PV contours defining the vortices boundary for $d / R=2.7$, and $\lambda_{s}=-0.02$ displayed at $t=0(a), 1.8(b), 3(c)$, and $6(d)$.

external field and the structure breaks back into two vortices. At this stage, the shear flow causes the vortices to separate and move away from one another. It is remarkable that this regime prevails for $\lambda_{s}=-0.04$ and -0.06 even for initial vortex separation distance $d / R$ down to 2 , where the two vortices initially touch. An illustration of such a interaction is proposed in figure 6 for $d / R=2.7$, and $\lambda_{s}=-0.02$. The initial phases are qualitatively similar to any merger situation. The main difference is the action of the adverse shear which tends to separate the vortices. But at $t=30$, the relative distance between the vortices is $d / R=13.9$. This regime is also prevalent for $\lambda_{s}=-0.08$ and in the range $d / R \in[2.4,2.5]$. A new regime is then however found for $d / R<2.4$. In these cases, the initial merger of the two vortices which creates a single central structure as before. The external shear is however intense enough, hence acting on a rapid enough time scale, to prevent the PV to rapidly re-organise itself into a dumbbell vortex. Hence the seed for the two vortices to form back does not have the time to develop. The single structure is simply sheared by the external field while the PV attempts to re-organise itself. An example of such a close range interaction in intense adverse shear is proposed in figure 7 for $d / R=2.1$ and $\lambda_{s}=0.08$.

For larger separation distances, the vortices are too far apart to merge and the vortices are pushed away from each other by the external adverse shear flow. An example of such a separation of the vortex pair is provided in figure 8 . The time evolution of the distance $d_{v}(t)$ between the vortex centroids is further illustrated in figure 3(a) (blue curve). When the vortices are far enough from each other, their mutual interaction weakens, and they are almost passively advected by external linear shear. This explains why, at later stage, the separation distance asymptotically tends to depend linearly of time. Meanwhile, the aspect ratio of the vortices oscillates quasi-periodically. The oscillation is triggered by the initial close range interaction of the vortices as the velocity field induced by the vortices on each other deform them. The oscillation persists as they move away from one another as there are no physical mechanism to damp the oscillation. The rotation of the vortex pair is illustrated in figure 9 by the time evolution of the angle $\theta=\arctan (\Delta x / \Delta y$ ), where $\Delta x$ (respectively $\Delta y$ ) is the difference between the $x$-coordinates (respectively $y$-coordinates) of the vortex centres. This shows that the vortex pair rotates only moderately while their shape oscillates. The increase 

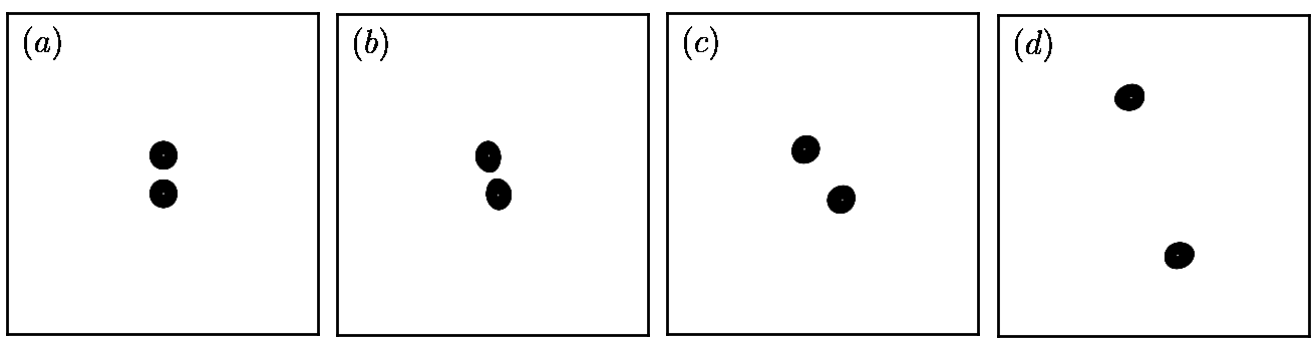

Figure 8. Illustration of a Separation ( $\diamond$ in figure 1): Top view on the PV contours defining the vortices boundary for $d / R=3$, and $\lambda_{s}=-0.02$ displayed at $t=0(a), 1.8(b), 10(c)$, and $30(d)$.

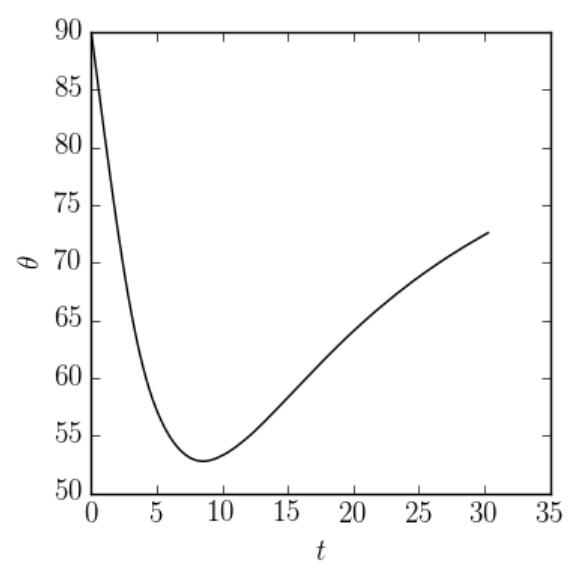

Figure 9. Time evolution of the angle $\theta$ of the axis joining the vortex centres to the $x$-axis for $d / R=3$, and $\lambda_{s}=-0.02$.

in $\theta$ at later stage is due to the separation of the vortices.

For more intense adverse shear (cases not shown) the vortices do not separate per se, but are simply sheared. Such regimes are also observed if two-dimensions and discussed in Trieling, Dam and van Heijst (2010) but are not relevant to the range of parameters used in the present study.

We note that the critical distances separating the different regimes decrease as the adverse shear is intensified. A simplified model of the problem consisting of a pair of point vortices in a linear shear is proposed in the Appendix A. It is a straightforward adaptation of the two dimensional model by Trieling, Dam and van Heijst (2010) to the three-dimensional QG situation. Although the point vortices do not deform and cannot merge, the model provides some indication on how the vortex centroid positions are affected by the shear. The model shows that for a cooperative shear, the vortices can get closer together. Indeed, the maximum separation distance between the point vortices along their periodic trajectory is obtained at $t=0$. Hence the merger of equivalent finite core vortices is facilitated. For a low adverse shear, the vortices are sightly pushed away from one another. In these cases the separation distance at $t=0$ is the minimum distance reached along the periodic trajectory of the point vortices. Beyond a threshold for the adverse shear, the point vortex trajectories are divergent, illustrating the separation of the vortices.

All these results provide some key dynamical elements to understand the interaction between the vortex pair and a surface buoyancy filament investigated in detail in the next section. 

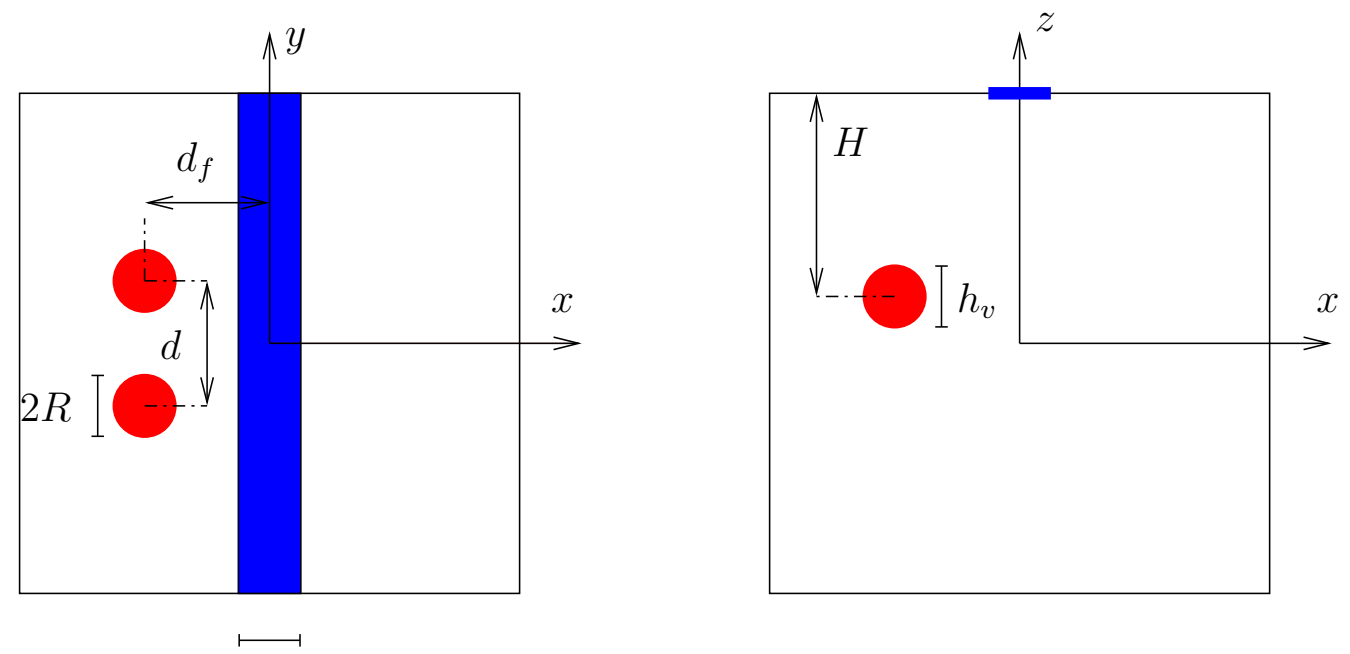

$2 a$

Figure 10. General geometry of the interaction between a pair of like-signed vortices (schematically represented in red) with a surface buoyancy filament (schematically represented in blue, colour online).

\section{Vortex merger near a surface buoyancy filament}

We now turn our attention to the interaction between the vortex pair and the surface buoyancy filament. The general geometry of the setup is described in figure 10. The surface filament is parallel to the $y$-direction, and located at $z=0$ with a half-width $a$. The vortices are located in the domain interior at a depth $z=-H$ from the surface. In this paper, we set $H=2 a$. The vortices have a height $h_{v}$ (in the stretched coordinate system), and a radius $R$. We restrict attention to spherical vortices, hence $h_{v} / R=2$. In all our numerical experiments we choose $a=R=0.25$, such that the vortices diameter equals the buoyancy filament width. A similar choice was used in Reinaud, Dritschel and Carton (2016) and Reinaud, Carton and Dritschel (2017). As in the previous section, the vortices are initially separated in the horizontal direction by a distance $d$. The line joining the vortex centres is initially parallel to the filament, and the vortex pair is located at a distance $d_{f}$ to the left of the axis $x=0$ which is the axis of symmetry of the filament. The surface buoyancy profile (normalised by the constant $N$ ) is defined at $t=0$ by

$$
\bar{b}(x)= \begin{cases}b_{m} \sqrt{1-\left(\frac{x}{a}\right)^{2}}, & \text { for }|x|<a, \\ 0, & \text { for }|x| \geq a .\end{cases}
$$

This distribution gives rise to a linear velocity profile with a shear rate $\alpha_{f}=-b_{m} / a$ (by construction) at the surface $z=0$ for $|x|<a$, as shown in figure 11(a). This means that, at the surface, the region $b_{m}>0$ corresponds to $\partial v / \partial x<0$. We define $\lambda_{f}=b_{m} /(q a)$ the ratio of the filament shear to the vortices PV. If $\lambda_{f}>0$, the filament is in adverse shear with the internal vortices, while if $\lambda_{f}<0$ the filament is in cooperative shear with the vortices. The linear stability of the filament alone is addressed in Reinaud, Dritschel and Carton (2016). The analysis shows that the the filament is unstable to perturbations with normalised longitudinal wavenumbers $k a \in(0,1.096)$. The peak instability is found at $k a=0.729$ with a non-dimensional growth rate $\sigma a / b_{m}=0.089$. Figure $11(b)$ shows the velocity induced by the unperturbed surface filament at the depth where the vortices are centred, $z=-H=-2 a$. We also see, from figure $11(b)$, that the unperturbed velocity profile induced by the filament alone at $z=-2 a$ can be split into 3 zones. Zone 2 is below the 

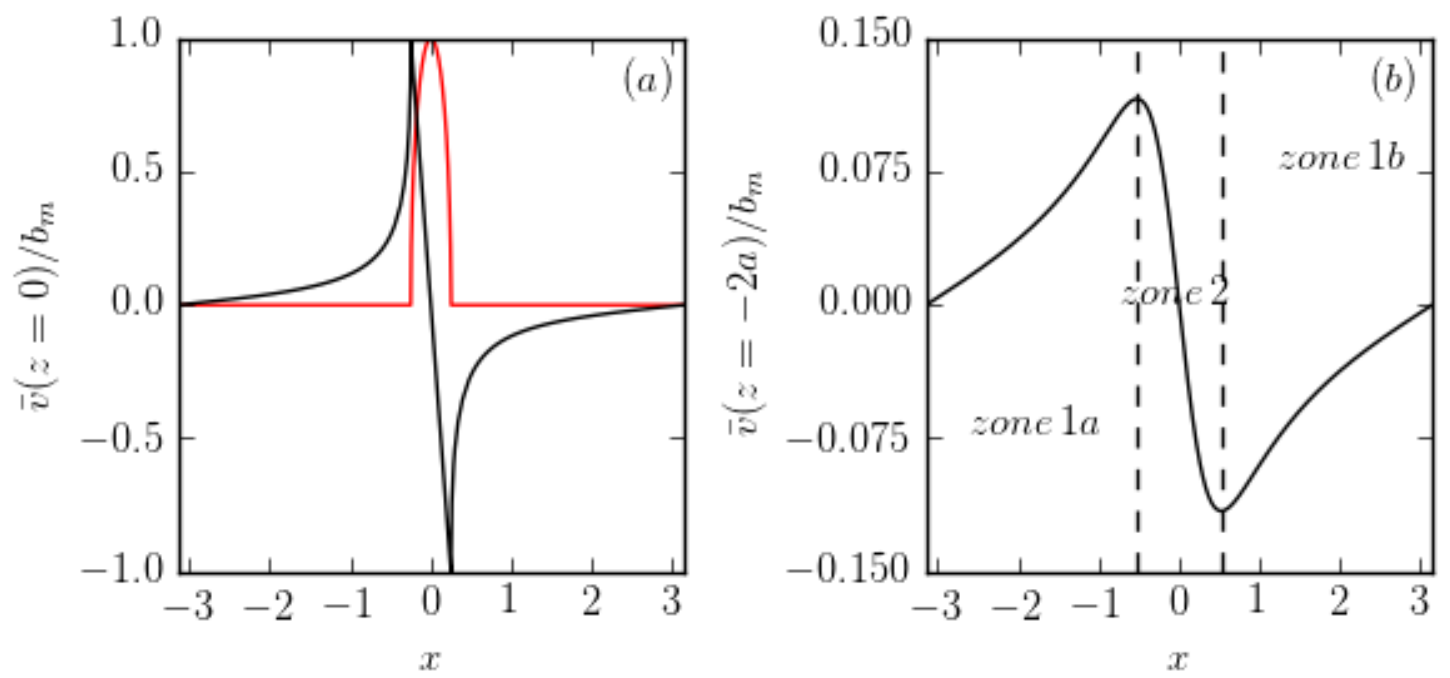

Figure 11. (a) Buoyancy distribution $\bar{b}$ (red) and velocity profile $\bar{v}$ (black) at the surface $z=0$, (b) velocity profile $\bar{v}$ at the depth $z=-2 a$ (colour online).

filament and zones $1 a$ and $1 b$ are on its left and right sides. Note that zone 2 is wider than the zone $\bar{b} \neq 0$ at $z=0$ as the influence of the filament spreads in the $x$-direction with depth. In zones $1 a$ and $1 b, \partial v / \partial x>0$ for $\lambda_{f}>0$, hence the filament in adverse shear with the vortices is in fact surrounded by two regions of cooperative shear. The situation is reverse when taking $\lambda_{f}<0$. It should be noted that the value of the shear at $z=-2 a$ is typically one order of magnitude less that the value at $z=0$. Hence the value we set of the shear experienced by the vortex in the following experiments is in fact consistent with the values used in Section 3.

Note that a surface filament also induces a vertical shear as the velocity it induces decreases with depth. The horizontal shear however appears to have a greater impact on the interaction regimes in our study.

We investigate the outcome of the interaction for various values of $d_{f}$ the initial distance between the vortex pair and the filament (which also dictates the zone of shear the vortices lie in initially), the shear ratio $\lambda_{f}$ and the relative distance $d / R$ between the vortices. Again we classify the outcome of the interactions. The categories are

(i) Merger (a • in figure 12). The two vortices merge directly.

(ii) Merger/Separation (a $\triangleleft$ in figure 12). The two vortices merge, then the structure breaks back into two vortices which separate.

(iii) Separation (a $\diamond$ in figure 12). The two vortices separate due to the external shear.

(iv) Merger/Break/Merger (a $\boldsymbol{\Delta}$ in figure 12). The vortices first merge, then break and merge back.

(v) No Merger (a $\square$ in figure 12). The vortices do not merge, and do not tend to separate.

(vi) Weak exchange $(\mathrm{a} \times$ in figure 12). The vortices touch creating a thin PV bridge between them which rapidly breaks.

(vii) Late Merger (a in figure 12). The two vortices merge but only after a transition period.

(viii) Separation/Alignment (a $\square$ in figure 12). Similar to a separation, but one of the vortices aligns with a surface billow.

We first consider $\lambda_{f}>0$. The outcome of the interactions are summarised in the top panels of figure 12. For $\lambda_{f}>0$, the filament is in adverse shear with the vortices. As mentioned previously, the filament is unstable and may break into billows at the surface. These billows 

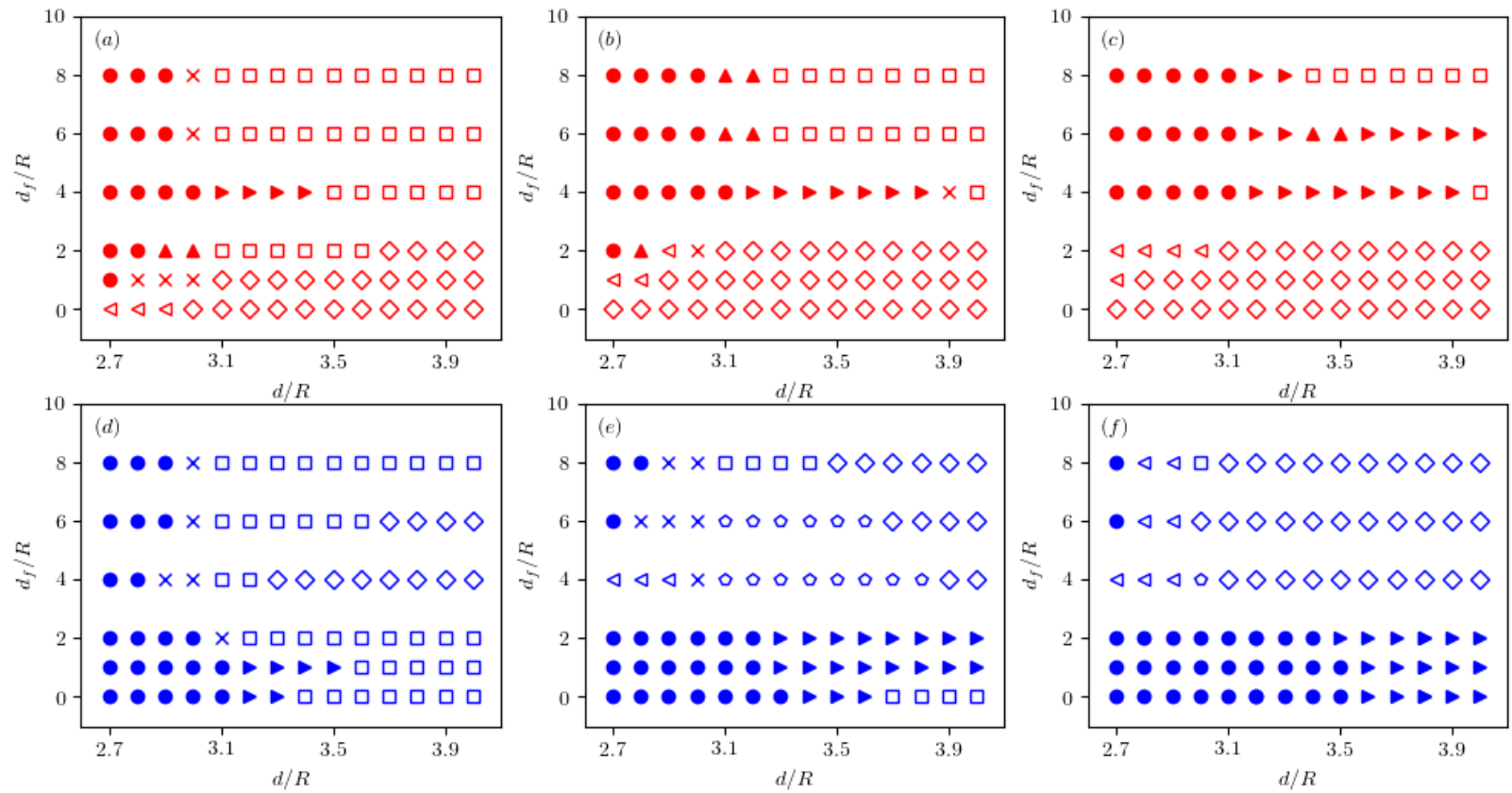

Figure 12. Outcome of the interaction between two spherical vortices of radius $R$, and PV $q$, initially distant of $d$, and at a distance $d_{f}$ from a surface buoyancy filament with $\lambda_{f}=b_{m} /(q a)=0.1(a), 0.4(b), 0.8(c),-0.1(d),-0.4(e),-0.8(f)$. - indicates Merger, $\triangleleft$ Merger/Separation, $\diamond$ Separation, $\Delta$ Merger/Break/Remerger, $\square$ No Merger, $\times$ Weak Exchange,

$\checkmark$ Late Merger, a $\nabla$ Merger/Break. $\square$ Separation/Alignment (colour online).

and the internal vortices are counter-rotating. For small values of $d_{f}$, the vortex pair is directly influenced by the adverse shear generated by the filament above it (zone 2 defined in figure 11). As seen in Section 3, an adverse shear impedes vortex merger. We see in figure 12 that for $d_{f}=0$, and $d / R=2.7$ the vortices do not merge for $\lambda_{f}=0.4$ and 0.8 . Recall that in absence of filament, vortices merge for this separation distance. The critical merger distance is in fact reduced when the adverse shear is applied. The panels $(a)$ and $(b)$ of figure 13 show a case for $d_{f}=0$ and $\lambda_{f}=0.4$ where the vortices merge. For this the separation has been reduced to $d / R=2.1$. It is also noteworthy that the filament has generated two billows which are away from the region where the merged vortex is. The depletion of buoyancy anomaly above a counter-rotating internal vortex is related to the way the vortex forces the filament. The shear induced by the vortex opposes the one induced by the filament, generating a stagnation point in the filament. Similar results were observed in the study of a filament interacting with a single vortex, see Reinaud, Dritschel and Carton (2016). For larger separation distances the vortex do not merge and are pulled apart when interacting with the filament, see the panels $(c)$ and $(d)$ of figure 13 for $d / R=3$. We also see that two large surface billows have symmetrically formed at the surface and interact more with the vortex closest to them. Figure 14 shows the time evolution of the vortices centres in these two cases. The panel $(a)$ of the figure shows the convergence of the centres leading to the merger for $d / R=2.1$ while the panels $(b)$ and $(c)$ illustrate the separation of the two vortices for $d / R=3$. In the special case $d_{f}=0$, the initial conditions are symmetric with respect to $z$-axis, hence the flow remains symmetric, within the accuracy of the calculation.

By taking $d_{f} \neq 0$, the initial symmetry is broken and this has an influence on the late evolution of the flow. We next illustrate the regimes of merger and of separation, discussed above for the symmetric case with $d_{f}=0$, in the case $d_{f} / R=2$. Results are presented in figure 15 for $\lambda_{f}=0.4$ and $d / R=2.7$ for an example of merger, and $d / R=3.1$ for an example of separation. Since $d_{f} / R=2$, the vortex pair is still mostly influenced by the adverse shear generated by the filament above. However, the shear is less intense than at 

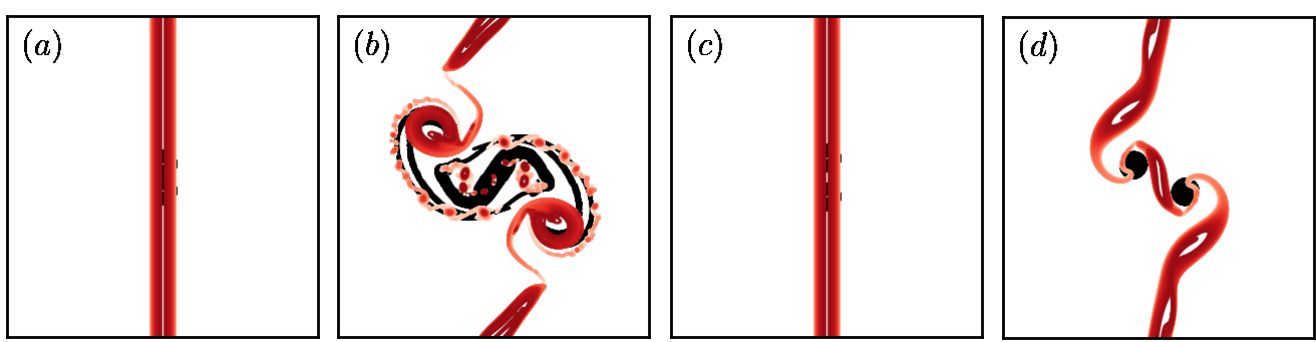

Figure 13. Top view on the buoyancy field (red) and the vortex boundaries (black) for $\lambda_{f}=0.4, d_{f}=0$, for a Merger at $d / R=2.1$ at $t=0,(a)$ and $t=30,(b)$, and a Separation at $d / R=3, t=0,(c)$ and $t=30,(d)$ (colour online).
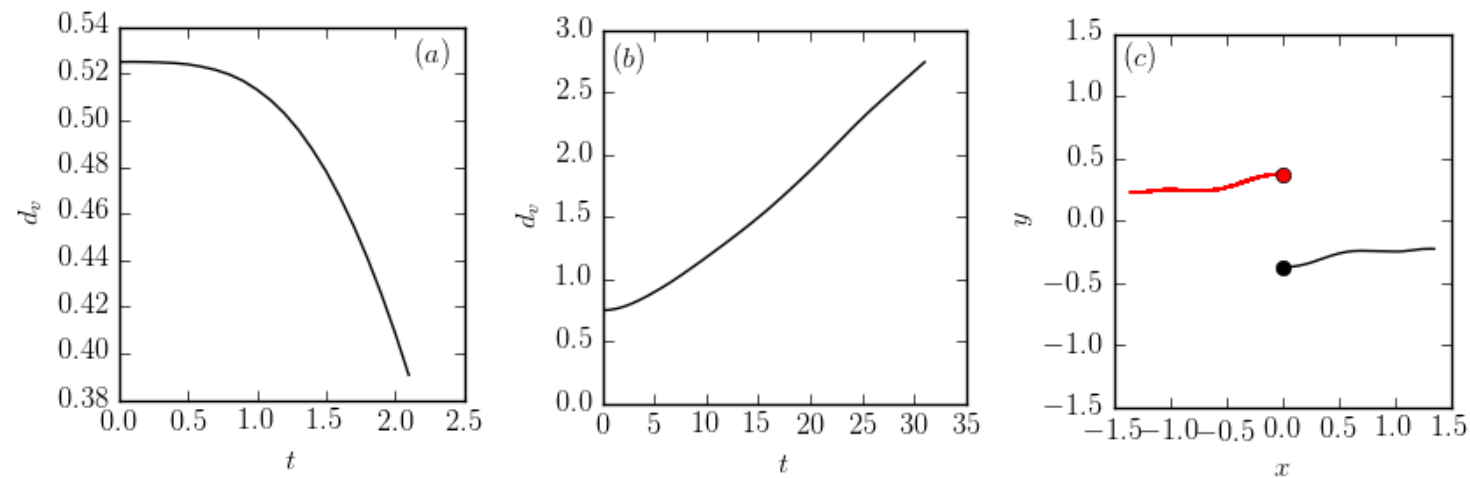

Figure 14. (a) Distance between the vortex centres $d_{v}$ vs time for $\lambda_{f}=0.4, d_{f}=0, d / R=2.1$. (b) Distance between the vortex centres $d_{v}$ vs time for $\lambda_{f}=-0.4, d_{f}=0, d / R=3$. (c) Trajectories of the vortex centres for $\lambda_{f}=-0.4$, $d_{f}=0, d / R=3$. The small circle indicates the initial positions of the vortices (colour online).

$d_{f}=0$. Hence the vortices can merge for $d / R=2.7$ as seen from the panels $(a)$ and $(b)$ of figure 15. The vortices force the filament which breaks asymmetrically into billows. One main buoyancy billow is generated ahead of the vortices. Recall that the billow and the vortices are counter-rotating for $\lambda_{f}>0$. This billow starts to interact with the merged vortices at depth to create a baroclinic dipole. This dipole starts to (slowly) propagate towards the "northwest' of the panel (i.e. $y>0, x<0)$, in contrast with the symmetric case $\left(d_{f}=0\right)$ where the merged vortical structure remained anchored at the centre of the domain. We also note that in both cases of merger, the surface billow tend to pull out large PV filaments from the merged structure, wrapping them around. This has the effect of limiting the 'efficiency' of the merger, that is the amount of PV gathered in a single main structure as a result of the interaction. For larger initial distance $d / R$, the vortices no longer merge, but separate. Again, since $d_{f} \neq 0$, the interaction is not symmetric. A generic example of such separation is given in the panels $(c)$ and $(d)$ of figure 15. Again, the filament break asymmetrically, with one large billow slightly closer to one of the vortices. The closest vortex has a stronger interaction with the opposite-signed billow, which results in the separation of the vortex pair. This time evolution of the distance between the vortex centres is presented in figure 16 for the the latter two cases. Panel $(c)$ of figure 16 illustrates the asymmetric trajectory of the vortices centres. In particular it clearly shows the consequence of the stronger interaction between the vortex initially located at $(0,+d / 2,-H)$ and the surface billow. As a results of its pairing with the opposite-signed billows the vortex moves further away from its initial position than the other vortex does.

For small value of $d_{f} / R$, and small value of $d / R$, yet beyond the full merger critical distance, a new regime of interaction is observed: the Merger/Separation. A similar regime is discussed in Section 3. Figure 17 illustrates such a case for $\lambda_{f}=0.4, d_{f} / R=1$ and $d / R=2.7$. In this example, the vortices initially merge but the shear induced by the filament is intense 

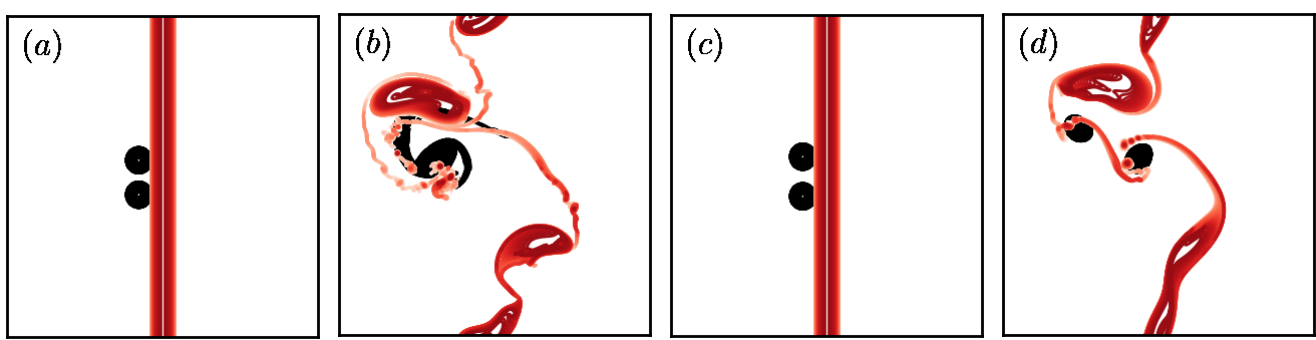

Figure 15. Top view on the buoyancy field (red) and the vortex boundaries (black) for $\lambda_{f}=0.4, d_{f} / R=2$, for a Merger $($ a $\bullet$ in figure 12) at $d / R=2.7$ at $t=0,(a)$ and $t=20,(b)$, and a Separation (a $\diamond$ in figure 12) at $d / R=3.1, t=0,(c)$ and $t=17,(d)$ (colour online).
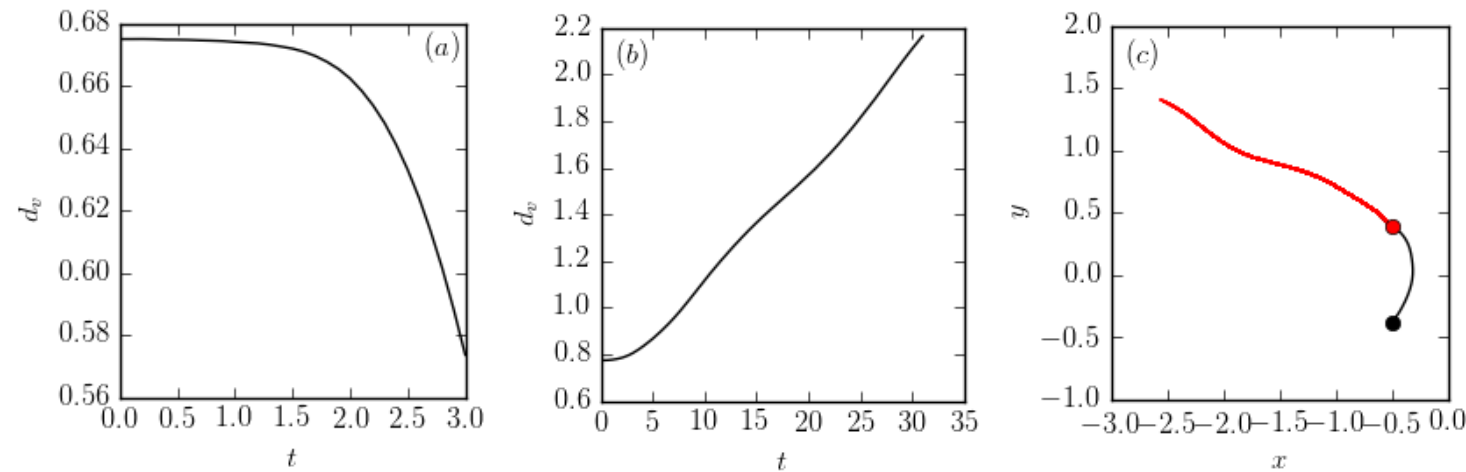

Figure 16. Time evolution of the distance $d_{v}$ between the two vortices for $\lambda_{f}=0.4, d_{f} / R=2$ and $d / R=2.7(a)$ and $d / R=3.1$ (b). Trajectory of the vortex centres $(c)$. The small circles indicate the centres position at $t=0$ (colour online).
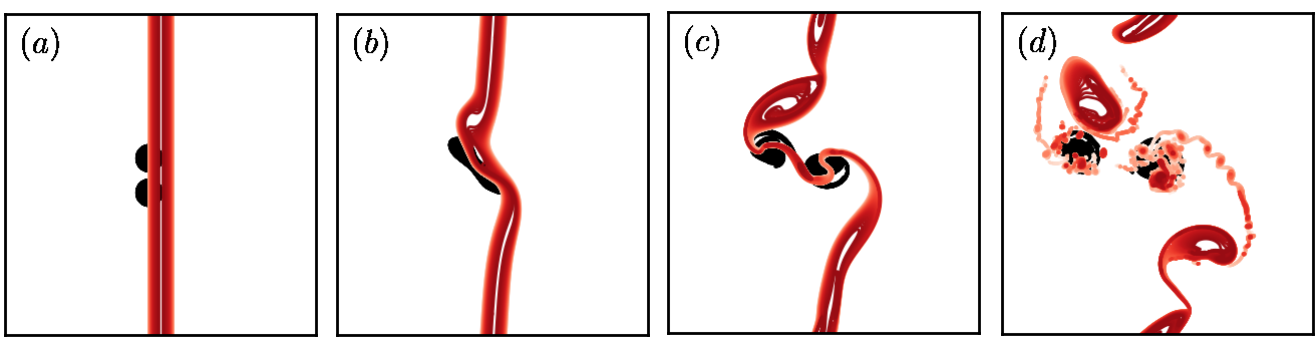

Figure 17. Top view on the buoyancy field (red) and the vortex boundaries (black) for $\lambda_{f}=0.4, d_{f} / R=1$, for a Merger/Separation (a $\triangleleft$ in figure 12) at $d / R=2.7$ at $t=0,(a), 3.6,(b), 10(c)$ and $t=30(d)$ (colour online).

enough to break the merged dumbbell structure. The resulting pair of vortices are then further separated by the interaction with the buoyancy billows formed at the surface. Again, due to the horizontal offset $d_{f} \neq 0$, the filament destabilises asymmetrically, forming a main billow which is closer to one vortex than the other. The billow and the closest vortex again couple as a baroclinic dipole which can move away from the other vortex, hence separating the vortex pair. The other vortex remains in a relatively quiet zone with only buoyancy debris and thin filaments in its vicinity. This interactions is generic of case with $\lambda_{f}>0$ and small values of $d_{f}$, where the dominant horizontal shear is adverse and when the vortices initially are relatively close to each other.

For larger values of $d_{f}$, the vortex pair is no longer in the adverse shear zone (zone 2 defined in figure 11) but in zone $1 a$ where the filament induces a cooperative shear. As a consequence there is a significant change in the interaction as the cooperative shear facilitates the merger. This is clearly shown in figure 12 where we observe regimes of merger for initial separation distances up to $d / R=3.1$ for $d_{f} / R=4$ and $\lambda_{f}=0.4$, and $d_{f} / R=4,6$ and 8 for $\lambda_{f}=0.8$. 

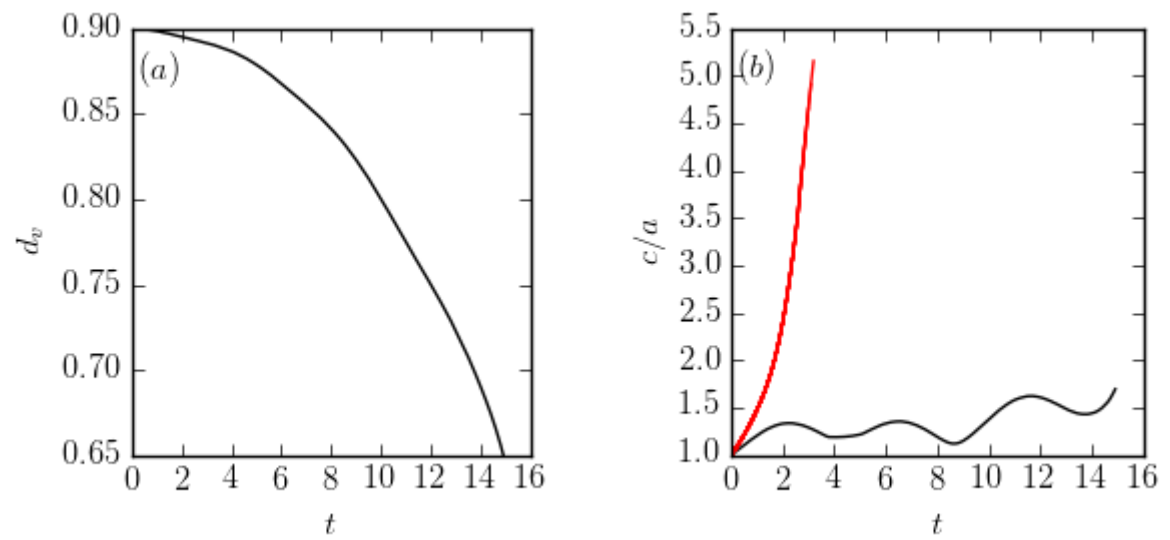

Figure 18. (a) Time evolution of the distance $d_{v}$ between the two vortices for late merger $\left(\downarrow\right.$ in figure 12) $\lambda_{f}=0.4$ $d_{f} / R=4$, and $d / R=3.6$. (b) Time evolution of one of the vortices aspect ratio $c / a$ for $\lambda_{f}=0.4, d_{f} / R=4$, and $d / R=3.6$ (black) and $d / R=2.7$ (red). The latter corresponds to a merger (a $\bullet$ in figure 12) (colour online).

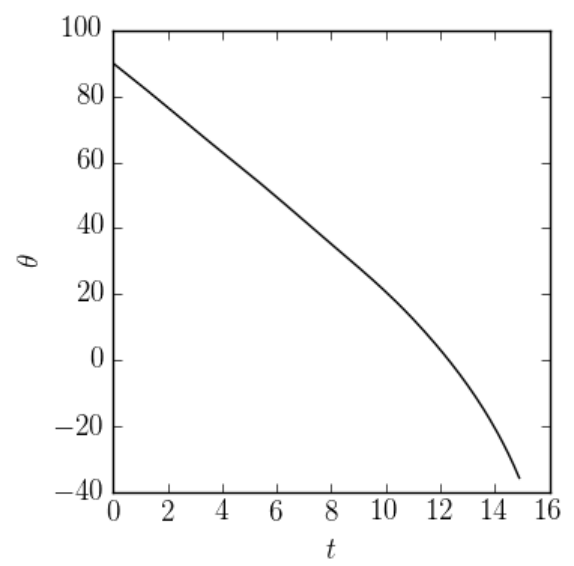

Figure 19. Time evolution the angle $\theta$ between the $x$-axis and the axis joining vortex centres for $\lambda_{f}=0.4, d_{f} / R=4$, and $d / R=3.6$.

This cooperative shear in zone $1 a$ is more intense near its edge with zone 2 , hence increasing $d_{f}$ further tends weakens the effect of the shear as the vortices are further away from the filament. This explains why the critical merger distance decreases as $d_{f} / R$ is increased beyond 4 .

For moderate values of $d_{f} / R$, such as $d_{f} / R=4$, where the cooperative shear is strong enough, we observe an extended range of moderate values of $d$, the separation distance between the vortices, for which the vortices merge late. As in the similar cases analysed in Section 3, the vortices are initially too far apart to merge, but the external shear brings the two vortices within a range where they can merge, while deforming them which arguably favours the merger by making the individual vortices less robust. As the vortices are pushed together, they pulse as can be seen from the oscillation of their aspect ratio $c / a$, where $c$ and $a$ are the largest and smallest semi-axis lengths of the best fitted ellipsoid. Figure 18 illustrates such an interaction by showing both the slower decrease of the vortex separation distance $d_{v}$ with time (compared to the monotonous elongation in a direct merger case), as well as the quasi-periodic oscillation of the vortex shape. In this example, $\lambda_{f}=0.4, d_{f} / R=4$, and $d / R=3.6$. We plot on figure 19 the angle $\theta$ showing the rotation of the vortex pair prior to merger, during the phase when the shape of the vortices oscillates. The vortex pair has rotated by an angle of nearly $126^{\circ}$ or $35 \%$ of a full rotation by $t=14.9$. 

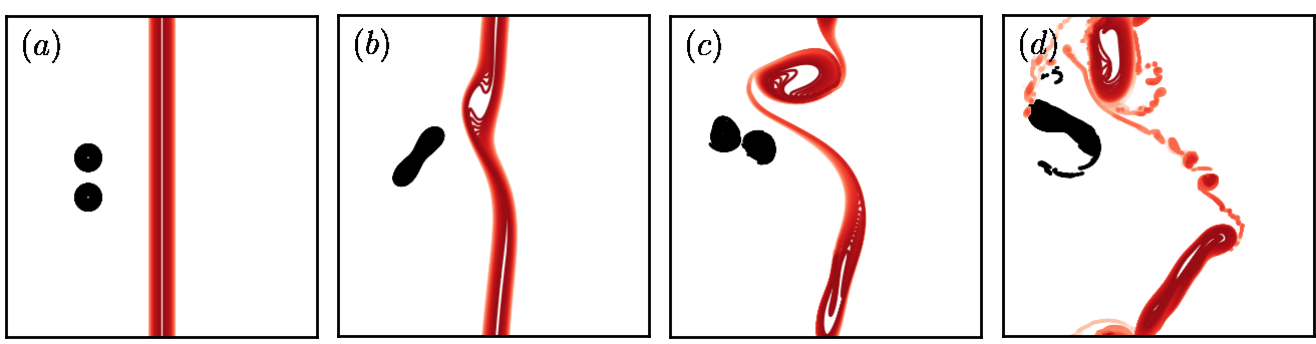

Figure 20. Top view on the buoyancy field (red) and the vortex boundaries (black) for $\lambda_{f}=0.4, d_{f} / R=6$, for a Merger/Break/Remerger (a $\boldsymbol{\Delta}$ in figure 12) at $d / R=3.1$ at $t=0,(a), 10,(b), 19(c)$ and $t=30(d)$ (colour online).

We also observe a few instances of regimes where the vortices first merge, and then the merged structure breaks but the vortices eventually merge back. They are mostly observed for initial separation distances larger than the critical distance but less than the lower bound of the range where vortices no longer merge at all. These are intermediate regimes where neither vortices form a close bound with the surface billows (hence the vortices are not moved away from one another). The vortices remain close to each other during the phase where they are separated, and the far field induced by the filament eventually push them to remerge. The regime is qualitatively similar to the equivalent regime of merger/break/remerger discussed in Section 3. A typical example of such interaction is presented in figure 20 for $\lambda_{f}=0.4$, relatively large $d_{f} / R=6$ and $d / R=3.1$.

Finally for large values of $d / R$, the vortices $(a)$ do not merge and separate if $d_{f} / R$ is small as the vortices experience an adverse shear induced by the filament, $(b)$ do not merge but do not separate for large values of $d_{f} / R$ as the filament is both distant and that the vortices experience a weak cooperative shear.

We next consider the outcome of the interaction between the vortex pair and surface filament in cooperative shear with $\lambda_{f}<0$. The outcome of the interactions for various values of $\lambda_{f}$, $d_{f}$ and $d$ are summarised in the bottom panels of figure 12. For small values of $d_{f}$, the vortex pair lies in zone 2 where they experience the effects of the cooperative shear and their merger is facilitated. The more intense the shear is, the largest the critical merger distance is. For example vortices may merge even if they are separated by $d / R=3.5$ for $\lambda_{f}=-0.8$ and $d_{f}=0$. A generic example of merger is presented in the panels $(a)$ and $(b)$ of figure 21 for $\lambda_{f}=-0.4, d_{f}=0$ and $d / R=2.7$. Note that a billow forms above the merged structure. Similar patterns were observed in Reinaud, Dritschel and Carton (2016) for the interaction of a single vortex and a filament in cooperative shear. The local rotation induced by the vortex seeds the formation of the billow in the filament. The rapid convergence of the vortex centres is illustrated in figure 22(a) from the rapid decrease of $d_{v}$, the distance separating them. For slightly larger separation distances, instances of later merger are observed. They are qualitatively similar to the other cases of late merger presented before and are not further illustrated here. For larger values of $d / R$ and $d_{f}=0$ the vortices do not merge. A large quasielliptical buoyancy billow forms at the surface and the vortex pair lies below it. A typical example is presented in figure 21 for $d / R=4, \lambda_{f}=-0.4$ and $d_{f}=0$. The time evolution of the distance $d_{v}$ between the vortices and the trajectory of their centres are given in figure 22 in panels $(b)$ and $(c)$ respectively. It should be noted that the trajectory of the vortices is, at least at these stages, almost elliptical with the vortices reaching the apogee of their trajectories near their initial position. This is consistent with the trajectory of the pair of point vortices in a uniform cooperative shear presented in the Appendix A.

Again, by taking $d_{f} \neq 0$, the symmetry seen in the two previous examples is broken. For small value of $d_{f}$ the vortex pair still lies in the zone where the shear is cooperative and merger is also facilitated. Again, a buoyancy billow forms at the surface ahead of the merging pair of vortices. But then, the co-rotating merged structure and the surface billow nearly align in the 


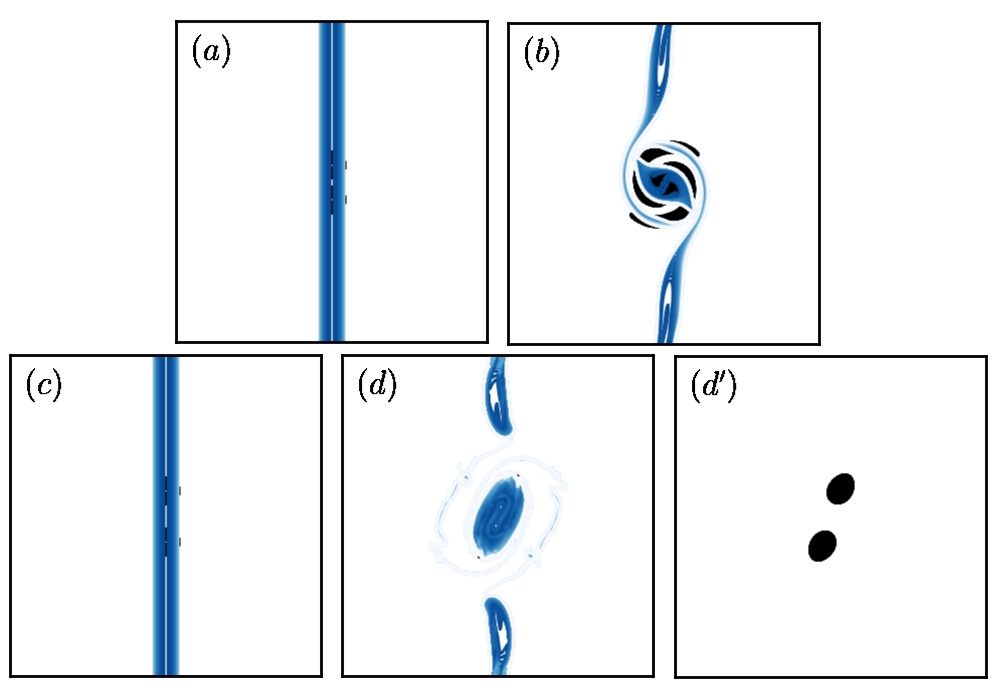

Figure 21. Top view on the buoyancy field (blue) and the vortex boundaries (black) for $\lambda_{f}=-0.4, d_{f}=0$ for an example of merger (a $\bullet$ in figure 12) with $d / R=2.7$ at $t=0,(a), 10,(b)$, for an example of no merger (a $\square$ in figure 12) for $d / R=4 t=0(c)$ and $17(d)$. Panel $\left(d^{\prime}\right)$ is the same as $(d)$ but we mask the filament to show the vortices (colour online).
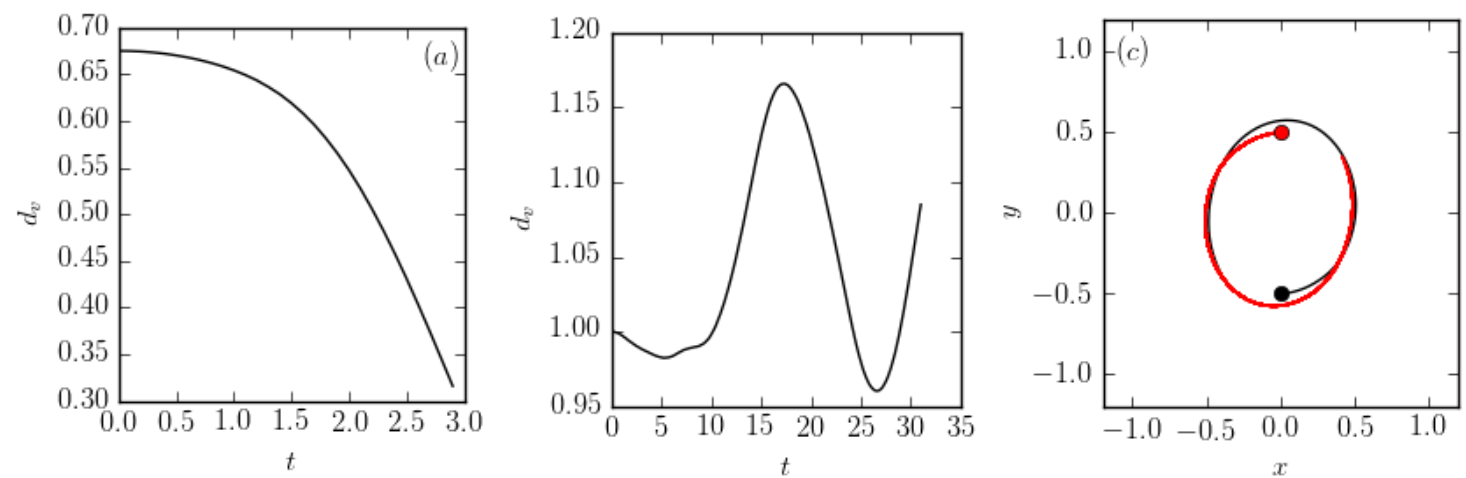

Figure 22. (a) Distance $d_{v}$ between the vortex centres vs time for $\lambda_{f}=-0.4, d_{f}=0$ and $d / R=2.7,(b): d / R=4$. $(c)$ Trajectories of the vortex centres for $\lambda_{f}=-0.4, d_{f}=0$ and $d / R=4$. The small circles indicate the initial position of the vortices (colour online).
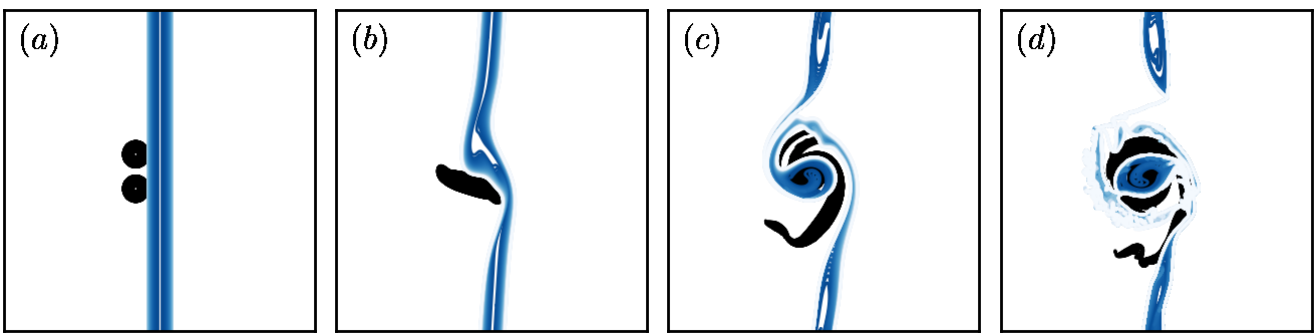

Figure 23. Top view on the buoyancy field (blue) and the vortex boundaries (black) for $\lambda_{f}=-0.4, d_{f} / R=2$, and $d / R=2.7$ for an example of merger (a $\bullet$ in figure 12) at $t=0,(a), 4,(b), 12(c)$, and $20(d)$ (colour online).

vertical direction. The alignment of co-rotating vortices has been, for example, documented in Polvani (1991) and more recently the alignment of buoyancy billow and a internal vortex has been shown in Reinaud, Dritschel and Carton (2017). An example of such interaction is presented in figure 23 for $\lambda_{f}=-0.4, d_{f} / R=2$, and $d / R=2.7$.

Rather different regimes are observed when $d_{f}$ is increased such that the vortex pair no longer lie in zone 2 at $t=0$ but in zone $1 a$. In these cases, the vortices experience an adverse 

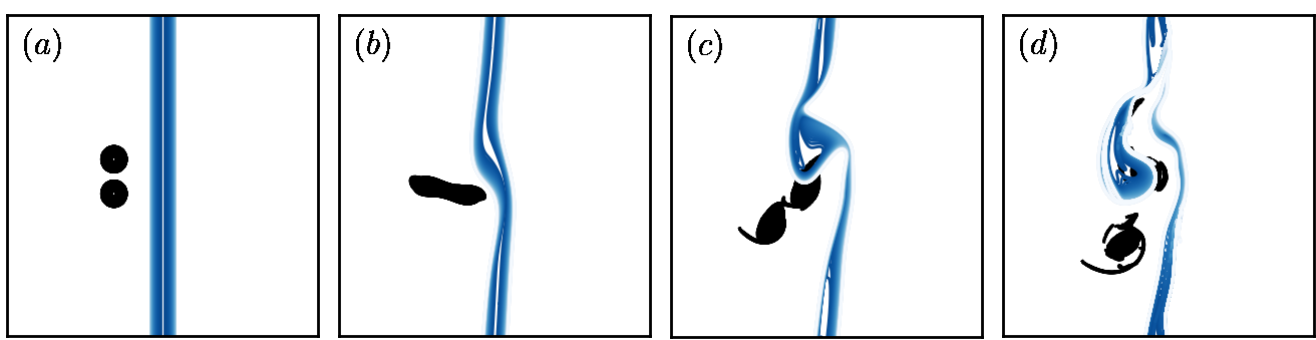

Figure 24. Top view on the buoyancy field (blue) and the vortex boundaries (black) for $\lambda_{f}=-0.4, d_{f} / R=4$, and $d / R=2.7$ for an example of merger (a $\bullet$ in figure 12) at $t=0,(a), 5,(b), 11(c)$, and $19(d)$ (colour online).
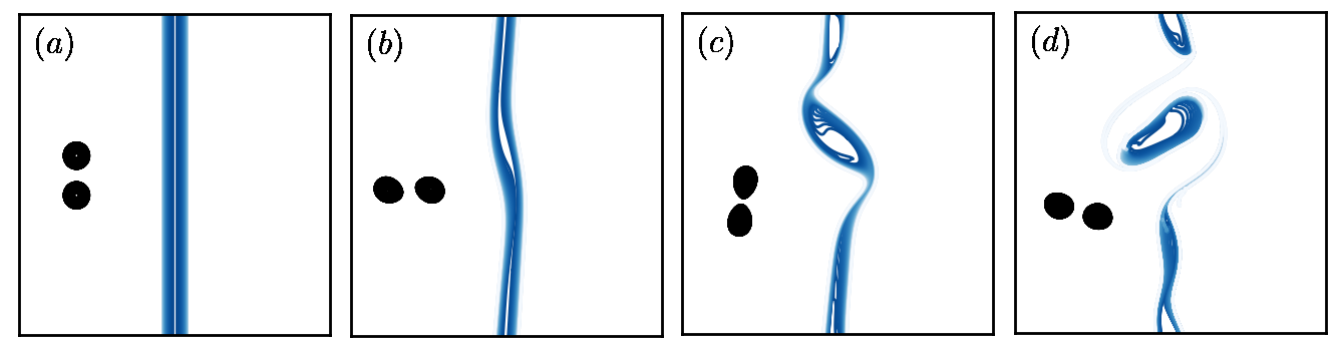

Figure 25. op view on the buoyancy field (blue) and the vortex boundaries (black) for $\lambda_{f}=-0.4, d_{f} / R=8$, and $d / R=3.1$ for an example of no merger (a $\square$ in figure 12 ) at $t=0,(a), 11,(b), 22(c)$, and $31(d)$ (colour online).

shear, even though, crucially, the billows which form at the surface are rotating in the same direction as the internal vortices. For very small separation distances, the vortices are still able to merge directly. Then, as seen from figure 12, for intermediate values of $d$ and $d_{f} / R=4,6$, and 8 we recover a regime where the vortices first merge, forming a dumbbell structure which breaks. The breaking of the structure is associated, and most likely favoured, by the alignment of one side of the merged dumbbell structure with a large surface billow which is seeded by the deformation induced by the vortices. Such an interaction is presented in figure 24 for $\lambda_{f}=-0.4, d_{f} / R=4$, and $d / R=2.7$.

Finally, for moderate to large values of $d_{f}$, when the vortices are initially in zone $1 a$, and for large values of $d$ the vortices no longer merge. In these cases, three slightly different regimes are observed, depending of the detailed interaction of the vortices with the filament and the billows which form from its destabilisation. In all cases, the filament destabilises and generate a large billow. If the vortices are close enough to each other, their mutual interaction can be stronger than their interaction with the billow. In such cases, the vortices do not separate. An example of such interaction is presented in figure 25 for $\lambda_{f}=-0.4, d_{f} / R=8$, and $d / R=3.1$. The time evolution of the distance between the vortices and their trajectories are presented in figure 26. Results indicate indeed that the vortices remains in the narrow range of separation distance.

The situation is slightly different for larger values of $\lambda_{f}$ or if the interaction between the vortices is weakened by increasing their initial separation distance $d / R$. Recall that the asymmetry of the situation $d_{f} / R$ means that one vortex in the pair is eventually closer to this billow, as seen in previous examples. For moderate values of $d_{f}$ this vortex is close enough to the billow to strongly interact with it, and the billow and the vortex align. This alignment provokes the separation of the vortex pair. An example of such an interaction is presented in the panels $(a)$ and $(b)$ of figure 27 for $\lambda_{f}=-0.4, d_{f} / R=4$, and $d / R=3.4$. If the vortices are further away from the filament, despite the fact that the interaction between one of the vortices with the surface billow becomes dominant over the vortices mutual interaction, this vortex is too far from the billow to completely align with it. This corresponds to a simple separation of the vortex pair. An example of such separation is presented in the panels $(c)$ and 

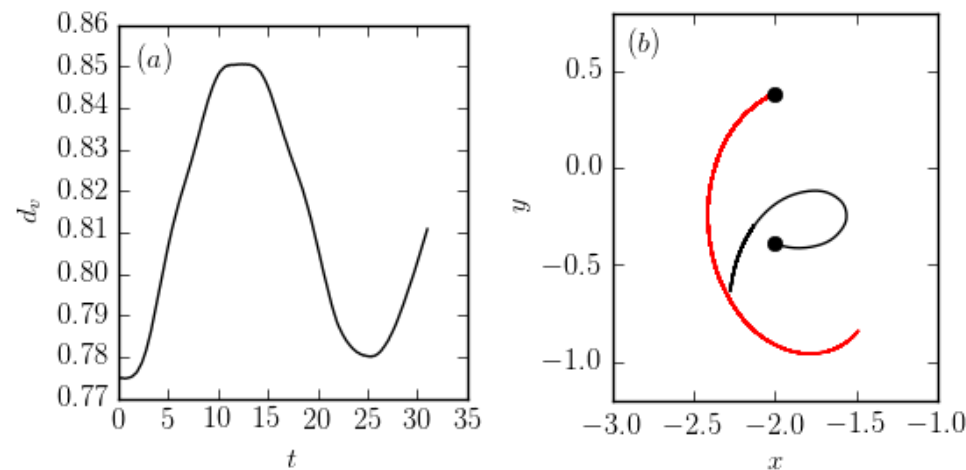

Figure 26. (a) Distance $d_{v}$ between the two vortex centres vs time for $\lambda_{f}=-0.4, d_{f} / R=8$, and $d / R=3.1$. (b) Trajectories of the vortex centres. The small circles indicate the initial position of the vortices (colour online).
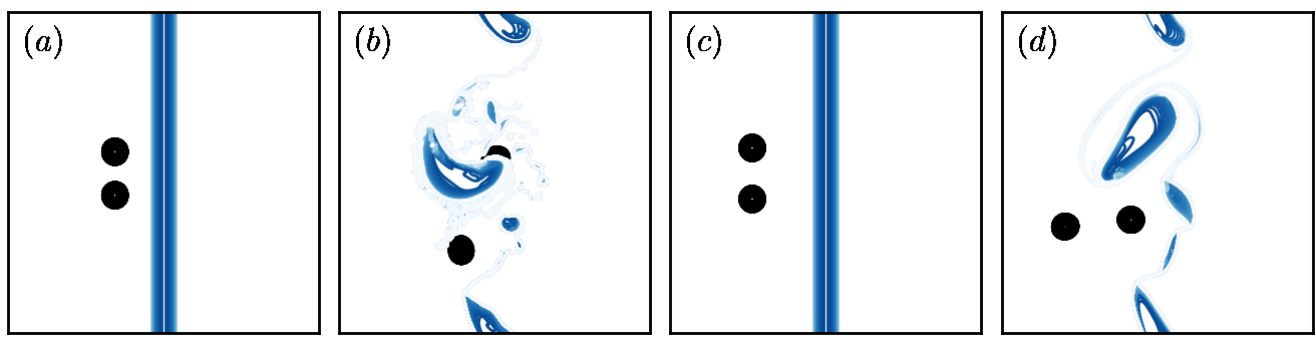

Figure 27. Top view on the buoyancy field (blue) and the vortex boundaries (black) for a separation/alignment $(\mathrm{a} *$ in figure 12) for $\lambda_{f}=-0.4, d_{f} / R=4$, and $d / R=3.4$ at $t=0,(a), 30,(b)$, and for a separation (a $\diamond$ in figure 12) for $\lambda_{f}=-0.4, d_{f} / R=6$, and $d / R=40(c)$, and $30(d)$ (colour online).
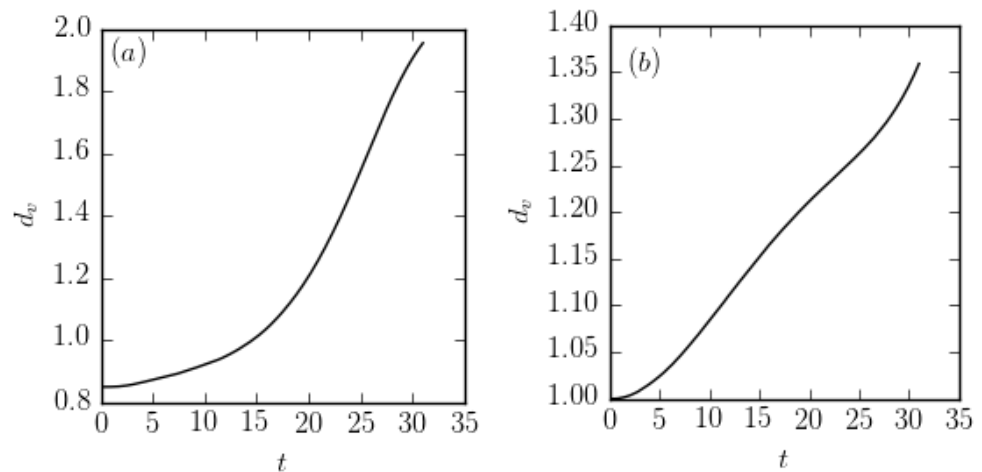

Figure 28. Separation distance between the two vortex centres $d_{v}$ vs time for $(a) \lambda_{f}=-0.4, d_{f} / R=4$, and $d / R=3.4$ and $(b) \lambda_{f}=-0.4, d_{f} / R=6$, and $d / R=4$

$(d)$ of figure 27 for $\lambda_{f}=-0.4, d_{f} / R=6$, and $d / R=4$. Finally, figure 28 shows the increases in time of the distance $d_{v}$ separating the two vortices confirming their separation in the two latter cases.

\section{Conclusion}

We have conducted the investigation of the interaction between two vortices in the vicinity of the surface buoyancy filament. To that end, we first focused on a further simplified situation where the vortices are subjected to a uniform horizontal shear. It is remarkable how relatively weak external shear, with a shear rate amounting for only a few percents of the vortices 
PV, drastically modifies the interaction. We confirm that a cooperative shear facilitates the merger. One key reason can be found in a simplified model which shows that a cooperative shear can bring the vortices closer together, see details in the Appendix A. On the other hand an adverse shear has the opposite effect. If the shear is weak, it impedes the merger by pushing the vortices apart without fully separating them. Intense adverse shear has the ability to separate the vortex pair completely.

These key dynamical effects proved essential to understand the interaction between the vortex pair and the filament. The filament indeed generates at depth regions of shear of both signs. Depending on the sign of the buoyancy anomaly and the relative position of the vortex pair with respect to the filament, the merger can be facilitated or impeded. Further to the effect of the shear, the fact that the filament destabilises into billows adds complexity to the problem. If the billow and the vortices are in co-rotation, the phenomenon of vortex alignment can occur. This can induce further separation of the vortex pair if the relative position of the vortices with respect to the billow is asymmetric. For symmetric interaction the alignment can in fact help the vortices remain in a bounded region are the three structures mutually interact. In the case where the billow and the vortices rotate in opposite direction, one vortex can pair with the billow to create a baroclinic dipole or 'hetonic' structure. Such structures are known to self-propagate in the oceans and provide a mechanism to transport heat in the oceans, see Hogg and Stommel (1985).

Vortices and pairs of vortices do not evolve in isolated environments, hence their mutual interaction is subject to external influences. We have considered in this paper a simple idealised situation where the external influence was due to a surface buoyancy filament. It should be noted that such filaments abound in the oceans. The study of the merger of like-signed vortices at depth under the influence of more realistic models of surface currents should be further investigated.

\section{References}

Bambrey, R.R., Reinaud, J.N. and Dritschel, D.G., Strong interactions between two co-rotating quasigeostrophic vortices. J. Fluid Mech., 2007, 592, 117-133.

Carton, X., Maze, G. and Legras, B., A two-dimensional vortex-merger in an external strain field. J. Turbul., $2002,3, \mathrm{~N} 45$.

Dijkstra, H.A., Dynamical oceanography, 2008 (Berlin Heidelberg: Springer-Verlag).

Dritschel, D.G., Contour surgery: a topological reconnection scheme for extended integrations using contour dynamics. J. Comput. Phys., 1988, 77, 240-266.

Dritschel, D.G., A general theory for two-dimensional vortex interactions. J. Fluid Mech., 1995, 293, 269-303.

Dritschel, D.G., Vortex merger in rotating stratified flows. J. Fluid Mech., 2002, 455, 83-101.

Dritschel, D.G. and Ambaum, M.H.P., A contour-advective semi-lagrangian numerical algorithm for simulating fine-scale conservative dynamical fields. Q. J. Roy. Meteor. Soc., 1997, 123, 1097-1130.

Dritschel, D.G. and Waugh, D.W., Qualification of the inelastic interaction of unequal vortices in twodimensional vortex dynamics. Phys. Fluids A, 1992, pp. 1737-1744.

Gula, J., Molemaker, M.J. and McWilliams, J.C., Submesoscale Cold Filaments in the Gulf Stream. J. Phys. Ocean., 2014, 44, 2617-2643.

Haidvogel, D., Beckman, A. and Hedström, K., Dynamical Simulations of Filament Formation and Evolution in the Coastal Transition Zone. J. Geophys. Res. Oceans, 1991, 96, 15017-15040.

Harvey, B.J. and Ambaum, M.H.P., Instability of surface-temperature filaments in strain and shear. Q. J. Roy. Meteor. Soc., 2010, 136, 1506-1515.

Hogg, N.G. and Stommel, H.M., The heton, an elementary interaction between discrete baroclinic geostrophic vortices, and its implications concerning eddy heat flow. Proc. R. Soc. Lond. A, 1985, 397, 1-20.

Iermano, I., Liguori, G., Iudione, D., BuongiornoÑardelli, B., Collela, S., Zingone, A., Saggioma, V. and Ribera d'Alcalà, M., Filament formation and evolution in buoyant coastal waters: Observation and modelling. Prog. Oceanogr., 2012, 106, 118-137.

Juckes, M., Instability of surface and upper-tropospheric shear lines. J. Atmos. Sci., 1995, 52, 3247-3262.

McWilliams, J.C., Gula, J., Molemaker, M.J., Renault, L. and Schchepetkin, A., Filament Frontogenesis by Boundary Layer Turbulence. J. Phys. Ocean., 2014, 45, 1988-2005.

Melander, M.V., Zabusky, N.J. and McWilliams, J.C., Symmetric vortex merger in two dimensions: causes and conditions. J. Fluid Mech., 1988, 195, 303-340. 
Overman II, E.A. and Zabusky, N.J., Evolution and merger of isolated vortex structures. Phys. Fluids, 1982, 25, 1297-1305.

Ozugurlu, E., Reinaud, J.N. and Dritschel, D.G., Interaction between two quasi-geostrophic vortices of unequal potential-vorticity. J. Fluid Mech., 2008, 597, 395-414.

Perrot, X., Reinaud, J.N., Carton, X. and Dritschel, D.G., Homostrophic Vortex Interaction under External Strain in a Coupled QG-SQG Model. Regul. Chaotic Dyn., 2010, 15, 66-83.

Polvani, L.M., Two-layer geostrophic vortex dynamics. Part 2. Alignment and two-layer V-states. J. Fluid Mech., 1991, 225, 241-270.

Reinaud, J.N., Carton, X. and Dritschel, D.G., Interaction between a quasi-geostrophic buoyancy filament and a heton. Fluids, 2017a, 2, 37.

Reinaud, J.N. and Dritschel, D.G., The merger of vertically offset quasi-geostrophic vortices. J. Fluid Mech., 2002, 469, 297-315.

Reinaud, J.N. and Dritschel, D.G., The critical merger distance between two co-rotating quasi-geostrophic vortices. J. Fluid Mech., 2005, 522, 357-381.

Reinaud, J.N., Dritschel, D.G. and Carton, X., Interaction between a surface quasi-geostrophic buoyancy filament and an internal vortex. Geophys. Astrophys. Fluid Dyn., 2016, 110, 464-490.

Reinaud, J.N., Dritschel, D.G. and Carton, X., Interaction between a surface quasi-geostrophic buoyancy anomaly jet and internal vortices. Phys. Fluids, 2017b, 29, 086603.

Richardson, P.L., Bower, A.S. and Zenk, W., A census of Meddies tracked by floats. Prog. Oceanogr., 2000, 45, 209250.

Richardson, P.L., McCartney, M.S. and Maillard, C., A search for meddies in historical data. Dyn. Atmos. Oceans, 1991, 15, 241265.

Scott, R.K., A scenario for finite-time singularity in the quasigeostrophc model. J. Fluid Mech., 2011, 687, 492-502.

Scott, R.K. and Dritschel, D.G., Numerical simulation of a self-similar cascade of filament instabilities in the surface quasigeostrophic system. Phys. Rev. Lett., 2014, 112, 144505.

Trieling, R.R., Dam, C.E.C. and van Heijst, G.J.F., Dynamics of two identical vortices in linear shear. Phys. Fluids, 2010, 22, 117104.

Vallis, G.K., Atmospheric and oceanic fluid dynamics: fundamentals and large-scale circulation, 2006 (Cambridge: Cambridge University Press).

von Hardenberg, J., McWilliams, J.C., Provenzale, A., Shchpetkin, A. and Weiss, J.B., Vortex merging in quasi-geostrohic flows. J. Fluid Mech., 2000, 412, 331353.

Waugh, D.W., The efficiency of symmetric vortex merger. Phys. Fluids A, 1992, pp. 1745-1758.

Zhang, Z., Wang, W. and Qiu, B., Oceanic mass transport by mesoscale eddies. Science, 2014, 345, 322-324.

\section{Appendix A: Point vortex model}

We propose in this appendix a simplified model of the vortex pair in uniform linear shear. We simply follow the calculation proposed by Trieiling, Dam and van Heisjt (2010) in twodimensions, transposed here to the three-dimensional QG situation. We model the two vortices by two singularities of strength $\kappa$ located respectively at $\left(x_{1}, y_{1}, z_{0}\right)$ and $\left(x_{2}, y_{2}, z_{0}\right)$ subject to a linear shear $v=\alpha x$. Initially we set $x_{1}=x_{2}=0$, and $y_{2}=-y_{1}=d / 2$. We denote $r(t)=\sqrt{\left(x_{2}-x_{1}\right)^{2}+\left(y_{2}-y_{1}\right)^{2}}$. The equation for the vortices trajectories are

$$
\begin{array}{r}
\frac{d x_{1}}{d t}=-\frac{\kappa}{4 \pi} \frac{y_{2}-y_{1}}{r^{3}}, \\
\frac{d x_{2}}{d t}=-\frac{\kappa}{4 \pi} \frac{y_{1}-y_{2}}{r^{3}}=-\frac{d x_{1}}{d t}, \\
\frac{d y_{1}}{d t}=-\frac{\kappa}{4 \pi} \frac{x_{2}-x_{1}}{r^{3}}+\alpha x_{1}, \\
\frac{d y_{2}}{d t}=-\frac{\kappa}{4 \pi} \frac{x_{1}-x_{2}}{r^{3}}+\alpha x_{2}=-\frac{d y_{1}}{d t}+\alpha\left(x_{1}+x_{2}\right) .
\end{array}
$$

We deduce that $X(t)=\frac{x_{1}+x_{2}}{2}$, and $Y(t)=\frac{y_{1}+y_{2}}{2}$, satisfy 


$$
\begin{gathered}
\frac{d X}{d t}=0, \\
\frac{d Y}{d t}=\alpha X .
\end{gathered}
$$

such that $X(t)=X_{0}$, a constant (zero here) and $Y(t)=\alpha X_{0} t+Y_{0}$. The Hamiltonian $H$ of the system is

$$
H=\frac{\kappa^{2}}{4 \pi} \frac{1}{\sqrt{\left(x_{2}-x_{1}\right)^{2}+\left(y_{2}-y_{1}\right)^{2}}}-\frac{\alpha \kappa}{2}\left(x_{1}^{2}+x_{2}^{2}\right),
$$

defined such that $d x_{i} / d t=\kappa^{-1} \partial H / \partial y_{i}$, and $d y_{i} / d t=-\kappa^{-1} \partial H / \partial x_{i}$. By conservation of $H$, the vortex trajectories are lines $H=H_{0}=\frac{\kappa^{2}}{4 \pi d}$, a constant. Note that for our initial condition $X_{0}=Y_{0}=0$ such that $X(t)=Y(t)=0$. The trajectories are of the form

$$
\frac{\xi^{2}+\eta^{2}}{d^{2}}=\left(\frac{1}{1+\mu(\xi / d)^{2}}\right)^{2}
$$

after simplification and using $\xi=\left(x_{2}-x_{1}\right), \eta=\left(y_{2}-y_{1}\right)$ and $\mu=d^{3} \alpha \pi / \kappa$. Note that since $X(t)=Y(t)=0$ for our initial conditions, $\xi=2 x_{2}=-2 x_{1}$, and $\eta=2 y_{2}=-2 y_{1}$. Note that in absence of external shear $(\alpha=\mu=0)$, we recover the expected circular trajectories for the vortex pair and $\xi^{2}+\eta^{2}=d^{2}$. Trajectories (contours of constant values for the Hamiltonian $H)$ are ilustrated in figure $\mathrm{A} 1$.

From equation A.8 is it clear that the diameter of the trajectory $\rho=\sqrt{\xi^{2}+\eta^{2}}$ is bounded from above by $d$ for $\mu>0$ as $1+\mu \xi^{2} \geq 1$. The diameter reaches $\rho=\eta=d$ for $\xi=0$. This is the case at $t=0$ in our set-up. The minimum is reached for $1+\mu \xi^{2}$ maximum, i.e. $\eta=0$. In this case this means that cooperative shear $(\mu>0)$ pushes the point vortices closer together for at initial stages $t>0$, facilitating the merger.

For $\mu<0$, there are two situations. In the first one, $1+\mu \xi^{2} \neq 0$ for all $\eta, \xi$. This is relevant for small value of $\mu$. Then $\rho$ is minimum for $\xi=0$ such as $t=0$. The vortices are therefore pushed away from each other at early stages. In the second situation, for larger values of $\mu$, the denominator $1+\mu \xi^{2}$ can reach zero, and then $\rho$ will diverge. In this case the pair of vortices separates. The threshold $\mu_{c}$ of $\mu$ between the two latter behaviours is $\mu_{c}=-4 / 27$ and corresponds to the value of $\mu$ for which the cubic equation in $\xi^{2}$ for $\eta=0$ deduced from equation A.8 has a triple real root. 


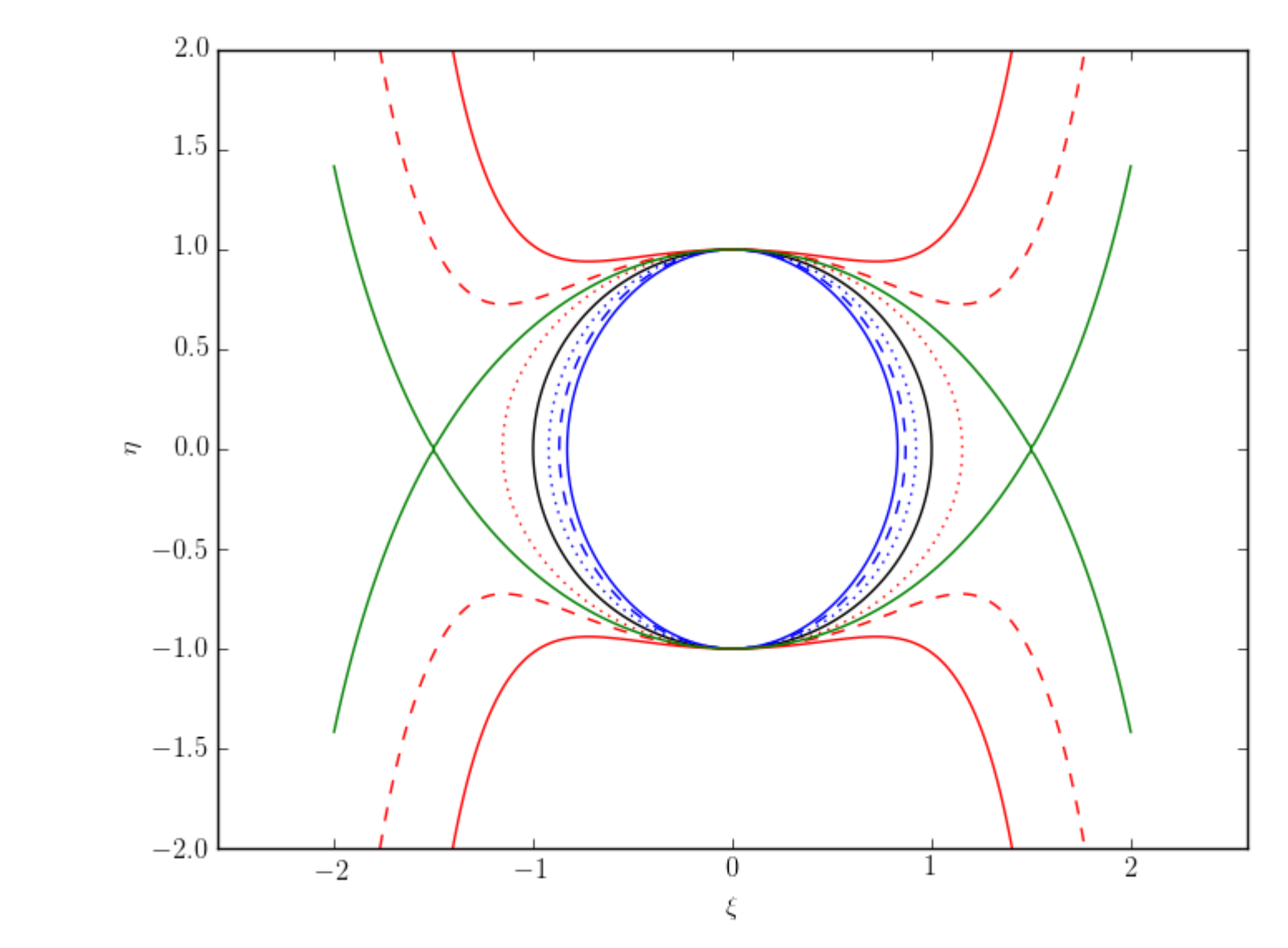

Figure A1. Contours of constant value for the Hamiltonian $H$ ('trajectories' in the $(\xi, \eta)$-plane) for $d=1$ and various

values of the parameter $\mu . \mu=0.1$ (blue, dotted), 0.2 (blue, dashed), 0.3 (blue, solid), 0 (black, solid), -0.1 (red, dotted), -0.2 (red, dotted) and -0.3 (red, solid). Critical value $\mu_{c}=-4 / 27$ (green, solid) (colour online).

(a) 